\title{
Ising critical behavior of inhomogeneous Curie-Weiss models and annealed random graphs
}

\author{
Sander Dommers ${ }^{(a)}$, Cristian Giardinà ${ }^{(b)}$, Claudio Giberti ${ }^{(\mathrm{c})}$, \\ Remco van der Hofstad ${ }^{(\mathrm{d})}$, Maria Luisa Prioriello ${ }^{(\mathrm{b}, \mathrm{d})}$. \\ (a) University of Bologna, Piazza di Porta San Donato 5, 40126 Bologna, Italy \\ (b) University of Modena and Reggio Emilia, via G. Campi 213/b, 41125 Modena, Italy \\ (c) University of Modena and Reggio Emilia, Via Amendola 2, 42122 Reggio Emilia, Italy \\ (d) Eindhoven University of Technology, P.O. Box 513, 5600 MB Eindhoven, The Netherlands
}

July 9,2018

\begin{abstract}
We study the critical behavior for inhomogeneous versions of the Curie-Weiss model, where the coupling constant $J_{i j}(\beta)$ for the edge $i j$ on the complete graph is given by $J_{i j}(\beta)=\beta w_{i} w_{j} /\left(\sum_{k \in[N]} w_{k}\right)$. We call the product form of these couplings the rank- 1 inhomogeneous Curie-Weiss model. This model also arises (with inverse temperature $\beta$ replaced by $\sinh (\beta)$ ) from the annealed Ising model on the generalized random graph. We assume that the vertex weights $\left(w_{i}\right)_{i \in[N]}$ are regular, in the sense that their empirical distribution converges and the second moment converges as well.

We identify the critical temperatures and exponents for these models, as well as a non-classical limit theorem for the total spin at the critical point. These depend sensitively on the number of finite moments of the weight distribution. When the fourth moment of the weight distribution converges, then the critical behavior is the same as on the (homogeneous) Curie-Weiss model, so that the inhomogeneity is weak. When the fourth moment of the weights converges to infinity, and the weights satisfy an asymptotic power law with exponent $\tau$ with $\tau \in(3,5)$, then the critical exponents depend sensitively on $\tau$. In addition, at criticality, the total spin $S_{N}$ satisfies that $S_{N} / N^{(\tau-1) /(\tau-2)}$ converges in law to some limiting random variable whose distribution we explicitly characterize.
\end{abstract}

\section{Introduction}

Universality is a key concept in the theory of phase transitions, with application to a large variety of physical systems. Informally, universality means that in the thermodynamic limit different systems show common properties close to criticality. The theory based on the renormalization group suggests that systems fall into universality classes, defined by the values of their critical exponents describing the nature of the singularities of measurable thermodynamic quantities at the critical point.

In the presence of heterogeneities, e.g. spin systems on random graphs used to model interaction on a network [1, 12, 13, 21] it is not clear a-priori to what extent universality applies. From the point of view of the structure of the network, emerging properties of real networks have been identified in several empirical studies in different contexts - social, information, technological and biological networks. Many of them are scale free, with a degree sequence obeying power-law distribution, and small world, with short graph-distance among vertices. As a consequence power-law random graphs, i.e., graph sequences where the fraction of nodes that have $k$ neighbors is proportional to $k^{-\tau}$ for some $\tau>1$, are often used as mathematical models for real-world networks. In this paper we investigate universality for spin system on power-law random graphs displaying phase transitions. 
The issue of universality is related to the network functionality. Indeed the occurrence of a thermodynamic phase transition is associated to a change in macroscopic properties of the networks, for instance the possibility to reach consensus in a social network can be related to the occurrence of a spontaneous magnetization. Thus the investigation of different universality classes for spin systems on random graphs is a relevant question with immediate practical relevance for the network functionality.

Due to the random environment, when considering the Ising model on the random graphs used to model real networks, a distinction is required between different averaging procedures. Two settings are often studied in the literature: the quenched measure (graph realizations are studied one-by-one so that they produce a random Boltzman-Gibbs measure) and the annealed measure (all graph realizations are considered at once and they give rise to a deterministic Boltzman-Gibbs measure). See [18, 7] for an extended discussion of the two settings.

In the paper [11] the quenched critical exponents have been rigorously analyzed for a large class of random graph models. More precisely in [11] it is proved that the critical exponent $\boldsymbol{\delta}$ (describing the behavior of the magnetization at the critical temperature as the external field vanishes), the exponent $\boldsymbol{\beta}$ (describing the behavior of the spontaneous magnetization as the temperature increases to the critical temperature) and the exponent $\gamma$ (describing the divergence of the susceptibility as the temperature decreases to the critical temperature) take the same values as the mean-field Curie-Weiss model whenever the degree distribution has a finite fourth moment. This includes for instance the case of the Erdős-Rényi random graph. For power law random graphs, it is proved that for $\tau>5$ the model is in the mean-field universality class, whereas the critical exponents are different from the mean-field values for $\tau \in(3,5)$.

In this paper we provide the analysis of the critical behavior but in the annealed setting. Our results are fully compatible with the universality conjecture. The annealed critical temperature is different (actually higher) than the quenched critical temperature, but the set of annealed critical exponents that can be rigorously studied are the same as the quenched critical exponents. In the annealed setting our results are stronger since we are able to show that $\gamma^{\prime}=\gamma$. Here $\gamma^{\prime}$ describes the divergence of the susceptibility as the temperature approaches to the critical temperature from below, and in the quenched setting we were able to show only that $\gamma^{\prime} \geq \gamma$.

A main difference between the quenched and annealed case is that while the analysis of the quenched measure could be done in great generality, the study of the annealed case is much harder. Indeed the results of [11] are valid for all graph sequences that are locally like a homogeneous random tree [2, 8, 9, 10, 22. and uniformly sparse. For the annealed setting it is not enough to control the behavior of the model on the typical graph realizations (namely rooted random trees). For the annealed measure one needs to study exponential functionals of the graphs, i.e., questions on large deviations of sparse random graphs that are largely unsolved. Thus we specialize our analysis of the annealed critical exponents to a particular class of random graphs models. This is given by the Generalized Random Graph models, also called inhomogeneous random graphs of rank-1 in the literature (see [20, 4] for a non-rigorous study).

By exploiting the factorization of the Gibbs measure and the edges independence we reduce the study of the annealed measure for the Ising model on the Generalized Random Graph to the analysis of an inhomogeneous Curie-Weiss model. As we shall see, for this model we are able to also study the properties at criticality. On a sequence of temperatures approaching the critical value, we prove the scaling limit for the properly renormalized total spin. As a result, our findings extend the analysis of the scaling limit of the standard Curie-Weiss model [15, 16, 14] and provides new asymptotic laws for the (properly renormalized) total spin. 


\section{Model definitions and results}

\subsection{Inhomogeneous Curie-Weiss model}

We start by defining the inhomogeneous Curie-Weiss model. This is a generalization of the classical CurieWeiss model in which the strength of the ferromagnetic interaction between spins is not spatially uniform. As the standard Curie-Weiss model, it is defined on the complete graph with vertex set $[N]:=\{1, \ldots, N\}$. See Table 1 at the end of the paper for a summary of the important notation used in this paper.

Definition 2.1 (Inhomogeneous Curie-Weiss model). Let $\sigma=\left\{\sigma_{i}\right\}_{i \in[N]} \in\{-1,1\}^{N}$ be spin variables. The inhomogeneous Curie-Weiss model, denoted by $\mathrm{CW}_{N}(\boldsymbol{J})$, is defined by the Boltzmann-Gibbs measure

$$
\mu_{N}(\sigma)=\frac{\mathrm{e}^{H_{N}(\sigma)}}{Z_{N}}
$$

where the Hamiltonian is

$$
H_{N}(\sigma)=\frac{1}{2} \sum_{i, j \in[N]} J_{i j}(\beta) \sigma_{i} \sigma_{j}+B \sum_{i \in[N]} \sigma_{i}
$$

and $Z_{N}$ is the normalizing partition function. Here $\beta$ is the inverse temperature, $B$ is the external magnetic field and $\mathbf{J}=\left\{J_{i j}(\beta)\right\}_{i, j \in[N]}$ are the spin couplings.

In the above, the interactions $J_{i, j}(\beta)$ might be arbitrary functions of the inverse temperature (in particular no translation invariance is required), provided that the thermodynamic limit is well-defined, i.e., the following limit defining the pressure exists and is finite,

$$
\phi(\beta, B):=\lim _{N \rightarrow \infty} \frac{1}{N} \log Z_{N}(\beta, B) .
$$

In the following we will restrict to the ferromagnetic version of the model, i.e., we will assume $J_{i j}(\beta)>0$. Since the coupling constants $\mathbf{J}=\left\{J_{i j}(\beta)\right\}_{i, j \in[N]}$ are positive and possibly different for different edges, we speak of an inhomogeneous Curie-Weiss model. We next state our hypotheses on the coupling variables. Each vertex $i \in[N]$ receives a weight $w_{i}$, We will take $\mathbf{J}=\left\{J_{i j}(\beta)\right\}_{i, j \in[N]}$ such that

$$
J_{i j}(\beta)=\frac{w_{i} w_{j}}{\ell_{N}} \beta, \quad \text { where } \quad \ell_{N}=\sum_{k \in[n]} w_{k} .
$$

In the case where $w_{i} \equiv 1$, our model reduces to the (homogeneous) Curie-Weiss model. We will call the coupling constants in (2.4) the rank-1 inhomogeneous Curie-Weiss model. In Section 2.3, we describe the assumptions that we make on the weight sequence $\boldsymbol{w}=\left(w_{i}\right)_{i \in[N]}$.

In [18] it is shown that the rank-1 inhomogeneous Curie-Weiss model arises in the study of the annealed Ising model with network of interactions given by the rank-1 inhomogeneous random graph, also called the generalized random graph, which we describe next.

\subsection{Generalized random graph}

In the generalized random graph [19, 5, each vertex $i \in[N]$ receives a weight $w_{i}$. Given the weights, edges are present independently, but the occupation probabilities for different edges are not identical, rather they are moderated by the weights of the vertices. We assume that the weights $\boldsymbol{w}=\left(w_{i}\right)_{i \in[N]}$ are strictly positive (there is no loss of generality in supposing this, since the vertices with zero weight will be isolated and can be removed from the network). 
Definition 2.2 (Generalized random graph). Denote by $I_{i j}$ the Bernoulli indicator that the edge between vertex $i$ and vertex $j$ is present and by $p_{i j}=\mathbb{P}\left(I_{i j}=1\right)$ the edge probability, where different edges are present independently. Then, the generalized random graph with vertex set $[N]$, denoted by $\operatorname{GRG}_{N}(\boldsymbol{w})$, is defined by

$$
p_{i j}=\frac{w_{i} w_{j}}{\ell_{N}+w_{i} w_{j}}
$$

where $\ell_{N}=\sum_{i=1}^{N} w_{i}$ is the total weight of all vertices.

We have now defined two classes of models that depend on vertex weights $\boldsymbol{w}=\left(w_{i}\right)_{i \in[N]}$. We next state the assumptions on these weights.

\subsection{Assumptions on the vertex weights}

We study sequences of inhomogeneous Curie-Weiss models and generalized random graphs as $N \rightarrow \infty$. For this, we need to assume that the vertex weight sequences $\boldsymbol{w}=\left(w_{i}\right)_{i \in[N]}$ are sufficiently nicely behaved. Let $U_{N} \in[N]$ denote a uniformly chosen vertex in $\operatorname{GRG}_{N}(\boldsymbol{w})$ and $W_{N}=w_{U_{N}}$ its weight. Then, the following condition defines the asymptotic weight $W$ and set the convergence properties of $\left(W_{N}\right)_{N \geq 1}$ to $W$ :

Condition 2.3 (Weight regularity). There exists a random variable $W$ such that, as $N \rightarrow \infty$,

(i) $W_{N} \stackrel{\mathcal{D}}{\longrightarrow} W$,

(ii) $\mathbb{E}\left[W_{N}^{2}\right]=\frac{1}{N} \sum_{i \in[N]} w_{i}^{2} \rightarrow \mathbb{E}\left[W^{2}\right]<\infty$,

where $\stackrel{\mathcal{D}}{\longrightarrow}$ denotes convergence in distribution. Further, we assume that $\mathbb{E}[W]>0$.

Note that, by uniform integrability, Condition 2.3 (ii) implies that also $\mathbb{E}\left[W_{N}\right]=\frac{1}{N} \sum_{i \in[N]} w_{i} \rightarrow \mathbb{E}[W]<$ $\infty$.

Condition 2.3 implies that the sequence $\left(\operatorname{GRG}_{N}(\boldsymbol{w})\right)_{N \geq 1}$ is a uniformly sparse tree-like graph with strongly finite mean and with asymptotic degree $D$ distributed as a mixed Poisson random variable,

$$
\mathbb{P}(D=k)=\mathbb{E}\left[\mathrm{e}^{-W} \frac{W^{k}}{k !}\right],
$$

see e.g., [19, Chapter 6].

Our results depend sensitively on whether the fourth moment of $W$ is finite. When this is not the case, then we will assume a power-law bound on the tail of the asymptotic weight:

Condition 2.4 (Tail of $W$ ). The random variable $W$ satisfies either of the following:

(i) $\mathbb{E}\left[W^{4}\right]<\infty$,

(ii) $W$ obeys a power law with exponent $\tau \in(3,5]$, i.e., there exist constants $C_{W}>c_{W}>0$ and $w_{0}>1$ such that

$$
c_{W} w^{-(\tau-1)} \leq \mathbb{P}(W>w) \leq C_{W} w^{-(\tau-1)}, \quad \forall w>w_{0} .
$$

To prove the results on the scaling limit at criticality we will strengthen our assumptions as follows:

Condition 2.5 (Tail of $W_{N}$ and deterministic sequences). The sequence of weights $\left(w_{i}\right)_{i \in[N]}$ satisfies either of the following:

(i) $\mathbb{E}\left[W_{N}^{4}\right]=\frac{1}{N} \sum_{i \in[N]} w_{i}^{4} \rightarrow \mathbb{E}\left[W^{4}\right]<\infty$, 
(ii) it coincides with the deterministic sequence

$$
w_{i}=c_{w}\left(\frac{N}{i}\right)^{1 /(\tau-1)},
$$

for some constant $c_{w}>0$ and $\tau \in(3,5)$.

We remark that the above deterministic sequence is $N$-dependent (we do not make this dependence explicit) and its limit $W$ satisfies (2.7) since $w_{i}=[1-F]^{-1}(i / N)$, where $F(x)=1-\left(c_{w} x\right)^{-(\tau-1)}$ for $w \geq c_{w}$. In the next section, we explain what the annealed measure of the Ising model on $\operatorname{GRG}_{N}(\boldsymbol{w})$ is.

\subsection{Annealed Ising Model}

We first define the annealed Ising model in general on finite graphs with $N$ vertices, then we specialize to $\mathrm{GRG}_{N}(\boldsymbol{w})$. We denote by $G_{N}=\left(V_{N}, E_{N}\right)$ a random graph with vertex set $V_{N}=[N]$ and edge set $E_{N} \subset V_{N} \times V_{N}$. We denote by $Q_{N}$ the law of the graphs with $N$ vertices.

Definition 2.6 (Annealed Ising measure). For spin variables $\sigma=\left(\sigma_{1}, \ldots, \sigma_{N}\right)$ taking values on the space of spin configurations $\Omega_{N}=\{-1,1\}^{N}$ the annealed Ising measure is defined by

$$
P_{N}(\sigma)=\frac{Q_{N}\left(\exp \left[\beta \sum_{(i, j) \in E_{N}} \sigma_{i} \sigma_{j}+B \sum_{i \in[N]} \sigma_{i}\right]\right)}{Q_{N}\left(Z_{G_{N}}(\beta, B)\right)},
$$

where

$$
Z_{G_{N}}(\beta, B)=\sum_{\sigma \in \Omega_{N}} \exp \left[\beta \sum_{(i, j) \in E_{N}} \sigma_{i} \sigma_{j}+B \sum_{i \in[N]} \sigma_{i}\right]
$$

is the partition function.

With abuse of notation in the following we use the same symbol to denote both a measure and the corresponding expectation.

Definition 2.7 (Annealed thermodynamic quantities). For a given $N \in \mathbb{N}$ we introduce the following thermodynamics quantities at finite volume:

(i) The annealed pressure:

$$
\psi_{N}(\beta, B)=\frac{1}{N} \log \left(Q_{N}\left(Z_{N}(\beta, B)\right)\right) .
$$

(ii) The annealed magnetization:

$$
M_{N}(\beta, B)=P_{N}\left(\frac{S_{N}}{N}\right),
$$

where the total spin is defined as

$$
S_{N}=\sum_{i \in[N]} \sigma_{i}
$$

(iii) The annealed susceptibility:

$$
\chi_{N}(\beta, B)=\frac{\partial}{\partial B} M_{N}(\beta, B) .
$$




\subsection{Annealed Ising Model on GRG}

We now specialize the previous definitions to the annealed Ising Model on the Generalized Random Graph. By assuming the probability $p_{i j}$ of each edge in $E_{N}$ is that given in (2.5), we can compute explicitly the average of the partition function (2.10). Indeed, recalling that $I_{i, j}$ is the indicator of the edge between vertex $i$ and $j$, we can write

$$
Q_{N}\left(Z_{N}(\beta, B)\right)=Q_{N}\left(\sum_{\sigma \in \Omega_{N}} \exp \left[\beta \sum_{i<j} I_{i j} \sigma_{i} \sigma_{j}+B \sum_{i \in[N]} \sigma_{i}\right]\right)
$$

and, by using the independence of the variables $I_{i, j}$, we compute [18] that

$$
Q_{N}\left(Z_{N}(\beta, B)\right)=C(\beta) \sum_{\sigma \in \Omega_{N}} \mathrm{e}^{B \sum_{i \in[N]} \sigma_{i}} \mathrm{e}^{\frac{1}{2} \sum_{i, j \in[N]} J_{i j}(\beta) \sigma_{i} \sigma_{j}},
$$

where $C(\beta)>0$ is a constant and the positive couplings $J_{i j}(\beta)$ are defined as

$$
J_{i j}(\beta)=\frac{1}{2} \log \left(\frac{\mathrm{e}^{\beta} p_{i j}+\left(1-p_{i j}\right)}{\mathrm{e}^{-\beta} p_{i j}+\left(1-p_{i j}\right)}\right) .
$$

The r.h.s. of (2.16) can be seen as the partition function of an inhomogeneous Curie-Weiss model with couplings $\mathbf{J}$ given by (2.17). Thus, the annealed Ising model on the $\operatorname{GRG}_{N}(\boldsymbol{w})$ is equivalent to such $\mathrm{CW}_{N}(\boldsymbol{J})$, i.e., the two measures coincide point-wise on the sample space. Our proof (see eq. (4.63) ) shows that the $J_{i j}(\beta)$ in (2.17) are close to the form in (2.4) with $\beta$ replaced by $\sinh (\beta)$, so that the study of the annealed generalized random graph reduces to the rank-1 ICW model. Preliminarily to the statement of our main results we recall the model solution given in [18]. By symmetry, we always take $B \geq 0$. We denote by $\beta_{c}$ the annealed critical inverse temperature defined as

$$
\beta_{c}:=\inf \left\{\beta>0: M\left(\beta, 0^{+}\right)>0\right\},
$$

where the spontaneous magnetization is given by

$$
M\left(\beta, 0^{+}\right)=\lim _{B \rightarrow 0^{+}} \lim _{N \rightarrow \infty} M_{N}(\beta, B) .
$$

Theorem 2.8 (Thermodynamic limit for annealed Ising on $\mathrm{GRG}_{N}(\boldsymbol{w})$ and for rank-1 $\mathrm{CW}_{N}(\boldsymbol{J})[18]$ ). Let $\left(G_{N}\right)_{N \geq 1}$ be a sequence of $\mathrm{GRG}_{N}(\boldsymbol{w})$ graphs satisfying Condition [2.3. Then,

(i) For all $0 \leq \beta<\infty$ and for all $B \in \mathbb{R}$, the annealed pressure exists in the thermodynamic limit $N \rightarrow \infty$ and is given by

$$
\psi(\beta, B):=\lim _{N \rightarrow \infty} \psi_{N}(\beta, B) .
$$

(ii) The magnetization per vertex exists in the limit $N \rightarrow \infty$ and is given by

$$
M(\beta, B):=\lim _{N \rightarrow \infty} M_{N}(\beta, B) .
$$

The limit value $M$ equals: $M(\beta, B)=\frac{\partial}{\partial B} \psi(\beta, B)$ for $B>0$, whereas $M=0$ in the region $0<\beta<\beta_{c}$, $B=0$. More explicitly, when $B>0$ or $B=0^{+}$and $\beta>\beta_{c}$

$$
M(\beta, B)=\mathbb{E}\left[\tanh \left(\sqrt{\frac{\sinh (\beta)}{\mathbb{E}[W]}} W z^{*}+B\right)\right],
$$

where $z^{*}=z^{*}(\beta, B)$ is the unique positive solution of the fixed point equation

$$
z=\mathbb{E}\left[\tanh \left(\sqrt{\frac{\sinh (\beta)}{\mathbb{E}[W]}} W z+B\right) \sqrt{\frac{\sinh (\beta)}{\mathbb{E}[W]}} W\right] .
$$


(iii) The annealed critical inverse temperature is given by

$$
\beta_{c}=\operatorname{asinh}(1 / \nu)
$$

where

$$
\nu=\frac{\mathbb{E}\left[W^{2}\right]}{\mathbb{E}[W]}
$$

(iv) The thermodynamic limit of susceptibility exists and is given by

$$
\chi(\beta, B):=\lim _{N \rightarrow \infty} \chi_{N}(\beta, B)=\frac{\partial^{2}}{\partial B^{2}} \psi(\beta, B) .
$$

(v) For the rank-1 inhomogeneous Curie-Weiss model $\mathrm{CW}_{N}(\boldsymbol{J})$, (i)-(iv) hold with $\beta$ replaced with $\operatorname{asinh}(\beta)$.

Theorem 2.8 shows that a phase transition exists for the annealed Ising model on the generalized random graph and the rank-1 inhomogeneous Curie-Weiss model. For the rank-1 inhomogeneous CurieWeiss model in the special case where $w_{i} \equiv 1$, Theorem 2.8 reproves the classical result for the Curie-Weiss model. When the weights are inhomogeneous, the critical value is instead given by $\beta_{c}=1 / \nu$.

Let us compare the annealed critical value in (2.24) to that in the quenched setting as derived in [17. There, it is proved that the quenched critical value $\beta_{c}^{\text {qu }}$ equals $\beta_{c}^{\text {qu }}=\operatorname{atanh}(1 / \nu)>\operatorname{asinh}(1 / \nu)=\beta_{c}$. Thus, the annealed critical value is smaller due to a collaboration of the Ising model and the graph properties.

In this paper, we analyze the block spin scaling limits at $\beta_{c}$ and we study the universality class of the model. For this, we define the annealed critical exponents analogous to the random quenched critical exponents as in [11]:

Definition 2.9 (Annealed critical exponents). The annealed critical exponents $\boldsymbol{\beta}, \boldsymbol{\delta}, \boldsymbol{\gamma}, \boldsymbol{\gamma}^{\prime}$ are defined by:

$$
\begin{aligned}
M\left(\beta, 0^{+}\right) & \asymp\left(\beta-\beta_{c}\right)^{\boldsymbol{\beta}}, & & \text { for } \beta \searrow \beta_{c} ; \\
M\left(\beta_{c}, B\right) & \asymp B^{1 / \delta}, & & \text { for } B \searrow 0 ; \\
\chi\left(\beta, 0^{+}\right) & \asymp\left(\beta_{c}-\beta\right)^{-\gamma}, & & \text { for } \beta \nearrow \beta_{c} ; \\
\chi\left(\beta, 0^{+}\right) & \asymp\left(\beta-\beta_{c}\right)^{-\gamma^{\prime}}, & & \text { for } \beta \searrow \beta_{c},
\end{aligned}
$$

where we write $f(x) \asymp g(x)$ if the ratio $f(x) / g(x)$ is bounded away from 0 and infinity for the specified limit.

We remark that, as is customary in the literature, we use the same letter for the inverse temperature $\beta$ and one of the magnetization critical exponent $\boldsymbol{\beta}$. In this paper they are distinguished by the use of the plain, respectively bold, character.

\subsection{Main results}

We start by proving that the annealed critical exponents for the magnetization and the susceptibility take the values conjectured in [20].

Theorem 2.10 (Annealed critical exponents). Let $\left(G_{N}\right)_{N \geq 1}$ be a sequence of $\mathrm{GRG}_{N}(\boldsymbol{w})$ graphs fulfilling Conditions 2.3 and 2.4. Then, the annealed critical exponents defined in Definition 2.9 using $\beta_{c}$ given in (2.24) exist and satisfy 


\begin{tabular}{c|cc} 
& $\tau \in(3,5)$ & $\mathbb{E}\left[W^{4}\right]<\infty$ \\
\hline $\boldsymbol{\beta}$ & $1 /(\tau-3)$ & $1 / 2$ \\
$\boldsymbol{\delta}$ & $\tau-2$ & 3 \\
$\gamma=\gamma^{\prime}$ & 1 & 1
\end{tabular}

For the boundary case $\tau=5$ there are the following logarithmic corrections for $\boldsymbol{\beta}=1 / 2$ and $\boldsymbol{\delta}=3$ :

$$
M\left(\beta, 0^{+}\right) \asymp\left(\frac{\beta-\beta_{c}}{\log 1 /\left(\beta-\beta_{c}\right)}\right)^{1 / 2} \quad \text { for } \beta \searrow \beta_{c}, \quad M\left(\beta_{c}, B\right) \asymp\left(\frac{B}{\log (1 / B)}\right)^{1 / 3} \text { for } B \searrow 0 .
$$

The same results hold for the rank-1 inhomogeneous Curie-Weiss model $\mathrm{CW}_{N}(\boldsymbol{J})$, the critical exponents being now defined using $\beta_{c}=1 / \nu$.

Remark 2.11 (Comparison to the Curie-Weiss model). For the rank-1 inhomogeneous Curie-Weiss model, we see that the inhomogeneity does not change the critical behavior when the fourth moment of the weight distribution remains finite, but it does when the fourth moment of the weight distribution increases to infinity. In the latter case, we call the inhomogeneity relevant.

Remark 2.12 (Comparison to the quenched case). In [11], the first two and fourth authors of this paper have shown that the same critical exponents hold for the quenched setting of the Ising model on power-law random graphs, such as $\mathrm{GRG}_{N}(\boldsymbol{w})$, under the assumptions in Conditions 2.3] and 2.4. In [11], however, we only managed to prove a one-sided bound on $\gamma^{\prime}$. Thus, our results show that for $\mathrm{GRG}_{N}(\boldsymbol{w})$ both the annealed and quenched Ising model have the same critical exponents, but a different critical value. This is a strong example of universality.

Remark 2.13 (Extension of $\boldsymbol{\gamma}=1$ ). The result $\boldsymbol{\gamma}=1$ holds under more general conditions, i.e., $\mathbb{E}\left[W^{2}\right]<$ $\infty$. See Theorem 3.6 below.

From the previous theorem we can also derive the joint scaling of the magnetization as $(\beta, B) \searrow\left(\beta_{c}, 0\right)$ :

Corollary 2.14 (Joint scaling in $B$ and $\left(\beta-\beta_{c}\right)$ ). For $\tau \neq 5$,

$$
M(\beta, B)=\Theta\left(\left(\beta-\beta_{c}\right)^{\boldsymbol{\beta}}+B^{1 / \boldsymbol{\delta}}\right),
$$

where $f(\beta, B)=\Theta(g(\beta, B))$ means that there exist constants $c_{1}, C_{1}>0$ such that $c_{1} g(\beta, B) \leq f(\beta, B) \leq$ $C_{1} g(\beta, B)$ for all $B \in(0, \varepsilon)$ and $\beta \in\left(\beta_{c}, \beta_{c}+\varepsilon\right)$ with $\varepsilon$ small enough. For $\tau=5$,

$$
M(\beta, B)=\Theta\left(\left(\frac{\beta-\beta_{c}}{\log 1 /\left(\beta-\beta_{c}\right)}\right)^{1 / 2}+\left(\frac{B}{\log (1 / B)}\right)^{1 / 3}\right) .
$$

Our second main result concerns the scaling limit at criticality. The next theorem provides the correct scaling and the limit distribution of $S_{N}$ at criticality (for a heuristic derivation of the scaling, see the discussion in Section 2.7). For $\operatorname{GRG}_{N}(\boldsymbol{w})$, we define the inverse temperature sequence

$$
\beta_{c, N}=\operatorname{asinh}\left(1 / \nu_{N}\right),
$$

where

$$
\nu_{N}=\frac{\mathbb{E}\left[W_{N}^{2}\right]}{\mathbb{E}\left[W_{N}\right]}
$$

so that $\beta_{c, N} \rightarrow \beta_{c}$ for $N \rightarrow \infty$. For rank-1 $\mathrm{CW}_{N}(\boldsymbol{J})$, we replace $\beta$ by $\operatorname{asinh}(\beta)$, so that $\beta_{c, N}=1 / \nu_{N}$. Our main result is the following: 
Theorem 2.15 (Non-classical limit theorem at criticality). Let $\left(G_{N}\right)_{N>1}$ be a sequence of $\mathrm{GRG}_{N}(\boldsymbol{w})$ graphs satisfying Conditions 2.3 and Condition 2.5 and let $\boldsymbol{\delta}$ have the respective values stated in Theorem [2.10. Then, there exists a random variable $X$ such that

$$
\frac{S_{N}}{N^{\boldsymbol{\delta} /(\boldsymbol{\delta}+1)}} \stackrel{\mathcal{D}}{\longrightarrow} X, \quad \text { as } N \rightarrow \infty
$$

where the convergence is w.r.t. the measure $P_{N}$ at inverse temperature $\beta_{c, N}=\operatorname{asinh}\left(1 / \nu_{N}\right)$ and external field $B=0$. The random variable $X$ has a density proportional to $\exp (-f(x))$ with

$$
f(x)= \begin{cases}\frac{1}{12} \frac{\mathbb{E}\left[W^{4}\right]}{\mathbb{E}[W]^{4}} x^{4} & \text { when } \mathbb{E}\left[W^{4}\right]<\infty, \\ \sum_{i \geq 1}\left(\frac{1}{2}\left(\frac{\tau-2}{\tau-1} x i^{-1 /(\tau-1)}\right)^{2}-\log \cosh \left(\frac{\tau-2}{\tau-1} x i^{-1 /(\tau-1)}\right)\right) & \text { when } \tau \in(3,5) .\end{cases}
$$

The same result holds for the rank-1 inhomogeneous Curie-Weiss model at its critical value $\beta_{c, N}=1 / \nu_{N}$.

We will see that in both the case where the fourth moment is finite as well as when it is infinite,

$$
\lim _{x \rightarrow \infty} \frac{f(x)}{x^{1+\delta}}=C,
$$

with

$$
C= \begin{cases}\frac{1}{12} \frac{\mathbb{E}\left[W^{4}\right]}{\mathbb{E}[W]^{4}} & \text { when } \mathbb{E}\left[W^{4}\right]<\infty, \\ \left(\frac{\tau-2}{\tau-1}\right)^{\tau-1} \int_{0}^{\infty}\left(\frac{1}{2} y^{-2 /(\tau-1)}-\log \cosh y^{-1 /(\tau-1)}\right) \mathrm{d} y & \text { when } \tau \in(3,5) .\end{cases}
$$

This result extends the non-classical limit theorem for the Curie-Weiss model to the annealed $\operatorname{GRG}_{N}(\boldsymbol{w})$ and the rank-1 $\mathrm{CW}_{N}(\boldsymbol{J})$.

\subsection{Discussion}

Random weights. Instead of choosing the weights $\boldsymbol{w}$ deterministically, one can also choose the weights i.i.d. according to some random variable $W$, with $\mathbb{E}\left[W^{4}\right]<\infty$. In this case, Condition 2.3 holds a.s. by the laws of large numbers. Hence, if $Q_{N}$ denotes the average over all graphs drawn according to the GRG conditioned on the weights, then our results also hold a.s. When in the annealing also the average over the weights is taken, then the model becomes unphysical, because the pressure becomes infinite as is proved in [18].

Critical exponents. Theorem 2.10 implies that the annealed exponents are the same as in the quenched case. Indeed, by (2.6), the condition $\mathbb{E}\left(W^{4}\right)<\infty$ is equivalent to $\mathbb{E}\left(K^{3}\right)<\infty$, where $K$ is the forward degree of the branching process describing the local structure of $\operatorname{GRG}_{N}(\boldsymbol{w})$. Thus the conditions in Theorem 2.10] defining the universality classes are the same as those in Theorem 2.8 in [11].

Scaling limit of block spin variable. In [18], it is proved that the classical central limit theorem for the total spin $S_{N}$ holds in the one-phase region of the annealed Ising model i.e.,

$$
\frac{S_{N}-P_{N}\left(S_{N}\right)}{\sqrt{N}} \stackrel{\mathcal{D}}{\longrightarrow} \mathcal{N}(0, \chi), \quad \text { w.r.t. } P_{N}, \quad \text { as } N \rightarrow \infty \text {. }
$$

In [17] we prove the analogous result in the quenched setting. More precisely, we prove (2.40) for the quenched measure in the quenched uniqueness regime for all random graphs that are locally tree-like. A prominent example is the $\mathrm{GRG}_{N}(\boldsymbol{w})$ as studied here. 
At criticality, i.e. for $(\beta, B)=\left(\beta_{c}, 0\right)$, the limit in (2.40) is no longer true. A scaling different from $\sqrt{N}$ has to be used to obtain the scaling limit, and also this limit is not a normal random variable. In [15, 16], Ellis and Newman prove that for the standard Curie-Weiss model

$$
\frac{S_{N}}{N^{3 / 4}} \stackrel{\mathcal{D}}{\longrightarrow} X, \quad \text { as } N \rightarrow \infty
$$

where $X$ is a random variable with density proportional to $\exp \left\{-\frac{1}{12} x^{4}\right\}$. We extend this result to the rank-1 inhomogeneous Curie-Weiss model, and thus to the annealed Ising model. We prove that the scaling with $N^{3 / 4}$ is also correct when $\mathbb{E}\left[W^{4}\right]<\infty$, but different for $\tau \in(3,5)$. Furthermore we show that when $\mathbb{E}\left[W^{4}\right]=\infty$, different asymptotic distributions arise in the scaling limit. We characterize them for the weight deterministic sequence (2.8) in which the weights follows a precise power-law. Such a sequence is rather generic in the sense that it produces an asymptotic weight that is also power-law distributed. The analysis shows that the fluctuations of the total spin decrease as the exponent $\tau$ becomes smaller and the distribution seen in the scaling limit has tails proportional to $\mathrm{e}^{-C x^{\tau-1}}$.

Heuristic for the scaling limit. To obtain a guess for the correct scaling, we can use the standard scaling relation between $\boldsymbol{\delta}$ and $\boldsymbol{\eta}$ as in [14]. On a box in the $d$-dimensional lattice with side lengths $n$, $[n]^{d} \subset \mathbb{Z}^{d}$, the exponent $\boldsymbol{\eta}$ satisfies

$$
P_{n}^{(d)}\left(S_{n}^{2}\right) \sim n^{d+2-\boldsymbol{\eta}},
$$

where $P_{n}^{(d)}$ is the expectation w.r.t. the Ising measure on this box and $S_{n}$ is the sum of all spins inside the box, where it should be noted that there are $n^{d}$ sites in the box. Hence, to compare this with our setting, we take $N=n^{d}$ and, with an abuse of notation, let $S_{n}=S_{N}$. If there is an exponent $\lambda$ such that $S_{N} / N^{\lambda}$ converges in distribution to a non-trivial limit, then it must also hold that $P_{N}\left(\left(S_{n} / N^{\lambda}\right)^{2}\right)=P_{N}\left(S_{n}^{2} / n^{2 d \lambda}\right)$ converges. Hence $S_{n}^{2} \sim n^{2 d \lambda}$, so that $d+2-\boldsymbol{\eta}=2 d \lambda$. The standard scaling relation $2-\boldsymbol{\eta}=d \frac{\boldsymbol{\delta}-1}{\boldsymbol{\delta}+1}$ [14] now suggests that we should choose

$$
\lambda=\frac{\delta}{\delta+1} .
$$

We prove that this is indeed the correct scaling and we also show that the tail of the density behaves like $\exp \left\{-C x^{\boldsymbol{\delta}+1}\right\}$ as is conjectured on $\mathbb{Z}^{d}$ (see [14, Section V.8]).

Near-critical scaling window. Theorem 2.15 is proved along the critical sequence $\beta_{c, N}$ approaching the critical inverse temperature $\beta_{c}$ in the limit $N \rightarrow \infty$. A different scaling limit might be obtained by working with a sequence near the critical one, the so-called near-critical window, i.e., $\beta_{c, N}+\Delta_{N}$ with $\Delta_{N} \rightarrow 0$ at an appropriate rate. As is argued in Section 4.5, it turns out that for the annealed Ising model the width $\Delta_{N}$ of the scaling window is $N^{-(\boldsymbol{\delta}-1) /(\boldsymbol{\delta}+1)}$ and the scaling limit differs by a quadratic term that appears in in the function $f(x)$ describing the density of $S_{N} / N^{\boldsymbol{\delta} /(\boldsymbol{\delta}+1)}$ in (2.37).

At criticality. As a consequence of the previous discussion, we also infer that if one works at critical inverse temperature $\beta_{c}$, the scaling limit that will be seen to depend on the speed at which $\nu_{N}$ approaches $\nu$. Indeed, from (2.24) and (2.34), one has $\beta_{c}-\beta_{c, N}=O\left(\nu-\nu_{N}\right)$. For a natural example given by the deterministic sequence in Condition 2.5 (ii) one has that when $\tau>5$ then $\nu-\nu_{N}=o\left(1 / N^{1 / 2}\right)$ and thus the limiting distribution does not change; on the contrary when $\tau \in(3,5]$ then $\nu-\nu_{N}=$ $\zeta N^{-(\boldsymbol{\delta}-1) /(\boldsymbol{\delta}+1)}(1+o(1))$ for some $\zeta \neq 0$, and thus the distribution changes since we are shifted in the near-critical window. See again Section 4.5 for more details. 
Organisation of this paper. In Section 3, we start by deriving the annealed critical exponents in Theorem 2.10. In Section 4, we prove our non-classical limit theorems at criticality in Theorem 2.15. We will prove our results only for the annealed $\operatorname{GRG}_{N}(\boldsymbol{w})$, since the proofs for the rank-1 inhomogeneous Curie-Weiss models are either identical, or simpler.

\section{Annealed critical exponents: proof of Theorem 2.10}

We follow a strategy similar to that in [11], although the proof in our case is a bit easier since the annealed magnetization is expressed in terms of the deterministic fixed point $z^{*}$ in (2.23), whereas in the quenched setting the magnetization is expressed in terms of a fixed point of a distributional recursion. The proof of Theorem 2.10 is split into Theorems 3.5 dealing with the exponents $\boldsymbol{\beta}$ and $\boldsymbol{\delta}$ (Section 3.1), Theorem 3.6 for the exponent $\gamma$ and Theorem 3.7 for the exponent $\gamma^{\prime}$ (Section 3.2). Some lemmas and propositions containing preliminary results are also stated and proved in Section 3.1.

Our analysis of the critical behavior crucially builds on the fixed point equation (2.23). We apply truncation arguments together with monotonicity (see the proof of Proposition 3.3 for a prototypical example). We rely on Taylor expansion properties for the fixed point $z^{*}$ in (2.23) as is customary for the Ising model. By truncation we mean that we decompose the range on integration of various expectations with respect to the limiting distribution $W$ according to the size of the fixed point $z^{*}$ and using asymptotics for truncated moments of $W$.

\subsection{Magnetization: critical exponents $\beta$ and $\delta$}

We start by showing that the phase transition is continuous.

Lemma 3.1 (Continuous phase transition). Let $\left(\left(\beta_{\ell}, B_{\ell}\right)\right)_{\ell \geq 1}$ be a sequence with $\beta_{\ell}$ and $B_{\ell}$ non-increasing, $\beta_{\ell} \geq \beta_{c}$ and $B_{\ell}>0$, and $\beta_{\ell} \searrow \beta_{c}$ and $B_{\ell} \searrow 0$ as $\ell \rightarrow \infty$. Then, the solution of (2.23) satisfies

$$
\lim _{\ell \rightarrow \infty} z^{*}\left(\beta_{\ell}, B_{\ell}\right)=0 \text {. }
$$

In particular,

$$
\lim _{B \searrow 0} z^{*}\left(\beta_{c}, B\right)=0, \quad \text { and } \quad \lim _{\beta \searrow \beta_{c}} z^{*}\left(\beta, 0^{+}\right)=0 .
$$

Proof. The existence of the limit (3.1) is a consequence of the monotonicity of $z^{*}(\beta, B)$ and the fact that $z^{*}(\beta, B) \geq 0$ for $B \geq 0$. Suppose that $\lim _{\ell \rightarrow \infty} z^{*}\left(\beta_{\ell}, B_{\ell}\right)=c>0$. Then, it follows from (2.23) and dominated convergence that

$$
c=\lim _{\ell \rightarrow \infty} z^{*}\left(\beta_{\ell}, B_{\ell}\right)=\mathbb{E}\left[\tanh \left(\sqrt{\frac{\sinh \left(\beta_{c}\right)}{\mathbb{E}[W]}} W c\right) \sqrt{\frac{\sinh \left(\beta_{c}\right)}{\mathbb{E}[W]}} W\right]<c \sinh \left(\beta_{c}\right) \nu=c,
$$

where we used that $\tanh (x)<x$ for $x>0$ and $\beta_{c}=\operatorname{asinh}(1 / \nu)$. This contradiction proves the lemma.

We next show that $z^{*}$ has the same scaling as we want to prove for $M(\beta, B)$ by proving the upper and lower bounds in Propositions 3.3 and 3.4 below. These then allow us to obtain the theorem. But first we state some properties for truncated moments of $W$ in the following lemma:

Lemma 3.2 (Truncated moments of $W$ ). Assume that $W$ obeys a power law for some $\tau>1$, see item (ii) in Condition 2.4. Then there exist constants $c_{a, \tau}, C_{a, \tau}>0$ such that, as $\ell \rightarrow \infty$,

$$
\left\{\begin{array}{l}
c_{a, \tau} \ell^{a-(\tau-1)} \\
c_{\tau-1, \tau} \log \ell
\end{array} \leq \mathbb{E}\left[W^{a} \mathbb{1}_{\{W \leq \ell\}}\right] \leq \begin{cases}C_{a, \tau} \ell^{a-(\tau-1)} & \text { when } a>\tau-1, \\
C_{\tau-1, \tau} \log \ell & \text { when } a=\tau-1 .\end{cases}\right.
$$


and, when $a<\tau-1$,

$$
\mathbb{E}\left[W^{a} \mathbb{1}_{\{W>\ell\}}\right] \leq C_{a, \tau} \ell^{a-(\tau-1)} .
$$

Proof. The proof is similar to that of [11, Lemma 3.4].

In the following we write $c_{i}, C_{i}, i \geq 1$ for constants that only depend on $\beta$ and on moments of $W$ and satisfy

$$
0<\liminf _{\beta \backslash \beta_{c}} c_{i}(\beta) \leq \limsup _{\beta \searrow \beta_{c}} c_{i}(\beta)<\infty,
$$

and the same holds for $C_{i}$. The constants $C_{i}$ appear in upper bounds and $c_{i}$ in lower bounds. Furthermore, we write $e_{i}, i \geq 1$ for error functions that depend on $\beta, B$ and on moments of $W$, and satisfy

$$
\limsup _{B \searrow 0} e_{i}(\beta, B)<\infty \quad \text { and } \quad \lim _{B \searrow 0} e_{i}\left(\beta_{c}, B\right)=0 .
$$

Here, the subscript $i$ is just a label for constants and error functions.

Further, we introduce the following notation that will be used extensively in the following:

$$
\alpha(\beta):=\sqrt{\frac{\sinh (\beta)}{\mathbb{E}[W]}} .
$$

Proposition 3.3 (Upper bound on $z^{*}$ ). Let $\beta \geq \beta_{c}$ and $B>0$. Then, there exists a $C_{1}>0$ such that

$$
z^{*} \leq \sqrt{\mathbb{E}[W] \sinh (\beta)} B+\sinh (\beta) \nu z^{*}-C_{1} z^{* \delta},
$$

where $\boldsymbol{\delta}$ takes the values as stated in Theorem 2.10. For $\tau=5$,

$$
z^{*} \leq \sqrt{\mathbb{E}[W] \sinh (\beta)} B+\sinh (\beta) \nu z^{*}-C_{1} z^{* 3} \log \left(1 / z^{*}\right) .
$$

Proof. We frequently use that $\tanh (B) \leq B$. A Taylor expansion around $x=0$ gives that, for some $\zeta \in(0, x)$

$$
\begin{aligned}
\tanh (x+B)= & \tanh (B)+\left(1-\tanh ^{2}(B)\right) x-\tanh (B)\left(1-\tanh ^{2}(B)\right) x^{2} \\
& \quad-\frac{1}{3}\left(1-\tanh ^{2}(\zeta+B)\right) x^{3}+\tanh (\zeta+B)\left(1-\tanh ^{2}(\zeta+B)\right) x^{3} \\
\leq & B+x-\frac{1}{3} x^{3}+\frac{4}{3} \tanh (x+B) x^{3}
\end{aligned}
$$

where we also used that $\tanh (x) \leq 1$. If we now assume that $x+B \leq \operatorname{atanh} \frac{1}{8}$, then

$$
\tanh (x+B) \leq B+x-\frac{1}{6} x^{3} .
$$

We apply this result to (2.23) where $x=\alpha(\beta) W z^{*}$, which we force to be at most atanh $\frac{1}{8}$ by introducing an indicator function as follows:

$$
\begin{aligned}
z^{*} \leq \mathbb{E}[ & \left.\left(B+\alpha(\beta) W z^{*}\right) \alpha(\beta) W\right] \\
+ & \mathbb{E}\left[\left\{\tanh \left(\alpha(\beta) W z^{*}+B\right)-\left(B+\alpha(\beta) W z^{*}\right)\right\} \alpha(\beta) W \mathbb{1}_{\left\{\alpha(\beta) W z^{*}+B \leq \operatorname{atanh} \frac{1}{8}\right\}}\right],
\end{aligned}
$$

since $\tanh (B+x) \leq B+x$. Hence, using (3.12),

$$
z^{*} \leq \sqrt{\mathbb{E}[W] \sinh (\beta)} B+\sinh (\beta) \nu z^{*}-\frac{1}{6} \alpha(\beta)^{4} \mathbb{E}\left[W^{4} \mathbb{1}_{\left\{\alpha(\beta) W z^{*}+B \leq \operatorname{atanh} \frac{1}{8}\right\}}\right] z^{* 3} .
$$


For $\mathbb{E}\left[W^{4}\right]<\infty$, this is indeed of the form (3.9) and we are done. If $\tau \in(3,5)$, then it follows from Lemma 3.2 that

$$
\mathbb{E}\left[W^{4} \mathbb{1}_{\left\{\alpha(\beta) W z^{*}+B \leq \operatorname{atanh} \frac{1}{8}\right\}}\right] \geq c_{4, \tau}\left(\frac{\alpha(\beta)}{\left(\operatorname{atanh} \frac{1}{8}-B\right)} z^{*}\right)^{\tau-5},
$$

which proves the proposition for $\tau \in(3,5)$. The proof for $\tau=5$ is similar and we omit it.

We now proceed with the lower bound:

Proposition 3.4 (Lower bound on $z^{*}$ ). Let $\beta \geq \beta_{c}$ and $B>0$. Then, there exists a $c_{1}>0$ such that

$$
z^{*} \geq \sqrt{\mathbb{E}[W] \sinh (\beta)} B+\sinh (\beta) \nu z^{*}-c_{1} z^{* \delta}-B e_{1},
$$

where $\boldsymbol{\delta}$ takes the values as stated in Theorem 2.10. For $\tau=5$,

$$
z^{*} \geq \sqrt{\mathbb{E}[W] \sinh (\beta)} B+\sinh (\beta) \nu z^{*}-c_{1} z^{* 3} \log \left(1 / z^{*}\right)-B e_{1} .
$$

Proof. As in (3.11) we can bound

$$
\tanh (x+B) \geq B+x-\frac{1}{3} x^{3}-B\left(B+B x+x^{2}\right),
$$

where we have used that $B-B^{2} \leq \tanh (B) \leq B$. For $\mathbb{E}\left[W^{4}\right]<\infty$, we can immediately use this to obtain

$$
z^{*} \geq \sqrt{\mathbb{E}[W] \sinh (\beta)} B+\sinh (\beta) \nu z^{*}-c_{1} z^{* 3}-B e_{1},
$$

where

$$
c_{1}=\frac{1}{3} \sinh ^{2}(\beta) \frac{\mathbb{E}\left[W^{4}\right]}{\mathbb{E}[W]^{2}}
$$

and

$$
e_{1}=B \sqrt{\mathbb{E}[W] \sinh (\beta)}+B \sinh (\beta) \nu z^{*}+\left(\frac{\sinh (\beta)}{\mathbb{E}[W]}\right)^{3 / 2} \mathbb{E}\left[W^{3}\right] z^{* 2} .
$$

All terms in $e_{1}$ indeed converge to 0 in the appropriate limit, because of Lemma 3.1 .

For $\tau \in(3,5)$, we rewrite $z^{*}$ as

$$
\begin{aligned}
z^{*}=\sqrt{\mathbb{E}[W] \sinh (\beta)} B+\sinh (\beta) \nu z^{*} & \\
& +\mathbb{E}\left[\left\{\tanh \left(\alpha(\beta) W z^{*}+B\right)-\left(B+\alpha(\beta) W z^{*}\right)\right\} \alpha(\beta) W\left(\mathbb{1}_{\left\{W \leq 1 / z^{*}\right\}}+\mathbb{1}_{\left\{W>1 / z^{*}\right\}}\right)\right] .
\end{aligned}
$$

The case where $W \leq 1 / z^{*}$ can be treated as above. This gives

$$
\begin{gathered}
\mathbb{E}\left[\left\{\tanh \left(\alpha(\beta) W z^{*}+B\right)-\left(B+\alpha(\beta) W z^{*}\right)\right\} \alpha(\beta) W \mathbb{1}_{\left\{W \leq 1 / z^{*}\right\}}\right] \\
\geq-\frac{1}{3} \sinh ^{2}(\beta) \frac{\mathbb{E}\left[W^{4} \mathbb{1}_{\left\{W \leq 1 / z^{*}\right\}}\right]}{\mathbb{E}[W]^{2}} z^{* 3}-B e_{2},
\end{gathered}
$$

where

$$
e_{2}=B \alpha(\beta) \mathbb{E}\left[W \mathbb{1}_{\left\{W \leq 1 / z^{*}\right\}}\right]+B \sinh (\beta) \frac{\mathbb{E}\left[W^{2} \mathbb{1}_{\left\{W \leq 1 / z^{*}\right\}}\right]}{\mathbb{E}[W]} z^{*}+\alpha(\beta)^{3} \mathbb{E}\left[W^{3} \mathbb{1}_{\left\{W \leq 1 / z^{*}\right\}}\right] z^{* 2}
$$

By Lemma 3.2,

$$
\mathbb{E}\left[W^{4} \mathbb{1}_{\left\{W \leq 1 / z^{*}\right\}}\right] \leq C_{4, \tau} z^{* \tau-5},
$$


so that indeed (3.23) is bounded from below by

$$
-c_{2} z^{* \tau-2}-B e_{2}
$$

with

$$
c_{2}=\frac{1}{3} \sinh ^{2}(\beta) \frac{C_{4, \tau}}{\mathbb{E}[W]^{2}} .
$$

Using Lemma's 3.1 and 3.2, one can also show that all terms in $e_{2}$ indeed converge to 0 in the appropriate limit.

It remains to bound the term where $W>1 / z^{*}$. For this we use that $\tanh (x+B) \geq 0$ :

$$
\begin{gathered}
\mathbb{E}\left[\left\{\tanh \left(\alpha(\beta) W z^{*}+B\right)-\left(B+\alpha(\beta) W z^{*}\right)\right\} \alpha(\beta) W \mathbb{1}_{\left\{W>1 / z^{*}\right\}}\right] \\
\geq-\sinh (\beta) \frac{\mathbb{E}\left[W^{2} \mathbb{1}_{\left\{W>1 / z^{*}\right\}}\right]}{\mathbb{E}[W]} z^{*}-B e_{3},
\end{gathered}
$$

where

By Lemma 3.2,

$$
e_{3}=\alpha(\beta) \mathbb{E}\left[W \mathbb{1}_{\left\{W>1 / z^{*}\right\}}\right]
$$

$$
\mathbb{E}\left[W^{2} \mathbb{1}_{\left\{W>1 / z^{*}\right\}}\right] \leq C_{2, \tau} z^{* \tau-3},
$$

again giving the right scaling. As a consequence (3.28) is bounded from below by $-c_{3} z^{* \tau-2}-B e_{3}$ with

$$
c_{3}=\frac{1}{3} \sinh (\beta) \frac{C_{2, \tau}}{\mathbb{E}[W]^{2}}
$$

Similarly,

$$
e_{3} \leq \alpha(\beta) C_{1, \tau} z^{* \tau-2}
$$

which indeed converges to 0 . We conclude that (3.16) holds with $c_{1}=c_{2}+c_{3}$ and $e_{1}=e_{2}+e_{3}$.

The upper and lower bounds on $z^{*}$ in the previous two propositions allow us to prove that the critical exponents take the values stated in Theorem 2.10 .

Theorem 3.5 (Values of $\boldsymbol{\beta}$ and $\boldsymbol{\delta}$ ). The critical exponents $\boldsymbol{\beta}$ and $\boldsymbol{\delta}$ equal the values as stated in Theorem 2.10 when $\mathbb{E}\left[W^{2}\right]<\infty$ and $\tau \in(3,5)$. Furthermore, for $\tau=5$ (2.31) holds.

Proof. Proof for exponent $\boldsymbol{\beta}$. We start by giving upper bounds on the magnetization. From (2.22) it follows that

$$
M(\beta, B)=\mathbb{E}\left[\tanh \left(\alpha(\beta) W z^{*}+B\right)\right] \leq B+\sqrt{\mathbb{E}[W] \sinh (\beta)} z^{*} .
$$

We first analyze $\boldsymbol{\beta}$ and hence take the limit $B \searrow 0$ for $\beta>\beta_{c}$. This gives

$$
M\left(\beta, 0^{+}\right) \leq \sqrt{\mathbb{E}[W] \sinh (\beta)} z_{0}^{*},
$$

where we write $z_{0}^{*}=\lim _{B \searrow 0} z^{*}$. Since $M\left(\beta, 0^{+}\right)>0$ by the definition of $\beta_{c}$, the same must be true for $z_{0}^{*}$. We will deal first with the cases $\tau \in(3,5)$ and $\mathbb{E}\left[W^{4}\right]<\infty$. Taking the limit $B \searrow 0$ in (3.9) and dividing by $z_{0}^{*}$, we get for $\tau \neq 5$

$$
C_{1} z_{0}^{* \boldsymbol{\delta}-1} \leq \sinh (\beta) \nu-1
$$

and hence, observing that $\boldsymbol{\beta}=1 /(\boldsymbol{\delta}-\mathbf{1})$,

$$
z_{0}^{*} \leq C_{1}^{-\boldsymbol{\beta}}(\sinh (\beta) \nu-1)^{\boldsymbol{\beta}} .
$$


From a Taylor expansion of $\sinh (\beta)$ around $\beta_{c}=\operatorname{asinh}\left(\mathbb{E}[W] / \mathbb{E}\left[W^{2}\right]\right)$ it follows that

$$
\sinh (\beta) \nu-1 \leq \cosh (\beta) \nu\left(\beta-\beta_{c}\right) .
$$

Hence,

$$
M\left(\beta, 0^{+}\right) \leq \sqrt{\mathbb{E}[W] \sinh (\beta)} C_{1}^{-\boldsymbol{\beta}}(\cosh (\beta) \nu)^{\boldsymbol{\beta}}\left(\beta-\beta_{c}\right)^{\boldsymbol{\beta}},
$$

so that it is easy to see that

$$
\limsup _{\beta \searrow \beta_{c}} \frac{M\left(\beta, 0^{+}\right)}{\left(\beta-\beta_{c}\right)^{\boldsymbol{\beta}}}<\infty .
$$

The lower bound can be obtained in a similar fashion. Starting from $\tanh x \geq x-x^{2}$ and taking the limit $B \searrow 0$ for $\beta>\beta_{c}$ in (2.22), we obtain

$$
M\left(\beta, 0^{+}\right) \geq \sqrt{\mathbb{E}[W] \sinh (\beta)} z_{0}^{*}-\sinh (\beta) \nu z_{0}^{* 2} .
$$

Again, starting from the lower bound (3.16), taking $B \searrow 0$ and dividing by $z_{0}^{*}$

$$
z_{0}^{*} \geq c_{1}^{-\boldsymbol{\beta}}(\sinh (\beta) \nu-1)^{\boldsymbol{\beta}},
$$

and, by a Taylor expansion around $\beta_{c}$,

$$
\sinh (\beta) \nu-1=\cosh \left(\beta_{c}\right) \nu\left(\beta-\beta_{c}\right)+O\left(\left(\beta-\beta_{c}\right)^{2}\right) .
$$

Using (3.36), (3.41) and (3.42) in (3.40) we obtain:

$$
\begin{aligned}
M\left(\beta, 0^{+}\right) & \geq \sqrt{\mathbb{E}[W] \sinh (\beta)} c_{1}^{-\boldsymbol{\beta}}\left[\cosh \left(\beta_{c}\right) \nu\left(\beta-\beta_{c}\right)+O\left(\left(\beta-\beta_{c}\right)^{2}\right)\right]^{\boldsymbol{\beta}} \\
& -\sinh (\beta) \nu C_{1}^{-2 \boldsymbol{\beta}}\left[\cosh \left(\beta_{c}\right) \nu\left(\beta-\beta_{c}\right)+O\left(\left(\beta-\beta_{c}\right)^{2}\right)\right]^{2 \boldsymbol{\beta}},
\end{aligned}
$$

which shows that also

$$
0<\liminf _{\beta \searrow \beta_{c}} \frac{M\left(\beta, 0^{+}\right)}{\left(\beta-\beta_{c}\right)^{\beta}},
$$

concluding the proof for the exponent $\boldsymbol{\beta}$ in the cases $\tau \in(3,5)$ and $\mathbb{E}\left[W^{4}\right]<\infty$. In the case $\tau=5$ we can prove the upper bound for $M\left(\beta, 0^{+}\right)$in a similar fashion, i.e., taking the limit $B \searrow 0$ for $\beta>\beta_{c}$ of (3.10) and dividing by $z_{0}^{*}$. This yields to

$$
z_{0}^{* 2} \leq \frac{\sinh (\beta) \nu-1}{C_{1} \log \left(1 / z_{0}^{*}\right)} \leq \frac{\cosh (\beta) \nu\left(\beta-\beta_{c}\right)}{C_{1} \log \left(1 / z_{0}^{*}\right)} \leq \widetilde{C} \frac{\left(\beta-\beta_{c}\right)}{\log \left(1 / z_{0}^{*}\right)}
$$

where (3.37) has been used in order to obtain the second inequality and $\cosh (\beta)$ has been bounded in a right neighborhood of $\beta_{c}$ to obtain the third inequality. Since $x \mapsto 1 / \log (1 / x)$ is increasing in $(0,1)$ and $z_{0}^{*} \leq C\left(\beta-\beta_{c}\right)^{\frac{1}{2}}$ for some $C>0,1$ form (3.45) we obtain:

$$
z_{0}^{* 2} \leq \frac{\widetilde{C}\left(\beta-\beta_{c}\right)}{C_{1} \log \left(1 /\left[C\left(\beta-\beta_{c}\right)^{1 / 2}\right]\right)} .
$$

The previous inequality together with (3.34), proves the upper bound

$$
\limsup _{\beta \searrow \beta_{c}} \frac{M\left(\beta, 0^{+}\right)}{\left(\frac{\beta-\beta_{c}}{\log \left(1 /\left(\beta-\beta_{c}\right)\right)}\right)^{1 / 2}}<\infty .
$$

\footnotetext{
${ }^{1}$ The proof of $z_{0}^{*} \leq C\left(\beta-\beta_{c}\right)^{\frac{1}{2}}$ can be obtained by rewriting (3.45) as $-z_{0}^{* 2} \log z_{0}^{* 2} \leq k\left(\beta-\beta_{c}\right)$, for some $k>0$. Since $w<-w \log w$ for $w<1 /$ e, we conclude that for $\beta-\beta_{c}>0$ small enough, the previous inequality gives $z_{0}^{* 2}<k\left(\beta-\beta_{c}\right)$.
} 
The lower bound can be obtained in the same way. Indeed, from (3.17) in the limit $B \rightarrow 0$, we obtain, for some positive constants $\widetilde{C}$ and $\widehat{C}$

$$
z_{0}^{* 2} \geq \frac{\sinh (\beta) \nu-1}{C_{1} \log \left(1 / z_{0}^{*}\right)} \geq \widetilde{C} \frac{\left(\beta-\beta_{c}\right)}{\log \left(1 / z_{0}^{*}\right)} \geq \widehat{C} \frac{\left(\beta-\beta_{c}\right)}{\log \left(1 /\left(\beta-\beta_{c}\right)\right)},
$$

where, once more, we have used that $x \mapsto 1 / \log (1 / x)$ is increasing in $(0,1)$ and the bound $z_{0}^{*} \geq C(\beta-$ $\left.\beta_{c}\right)^{1 /(2-\varepsilon)}$ for some $C>0$ and any $0<\varepsilon<22$ The previous inequality plugged in (3.40) gives

$$
\liminf _{\beta \backslash \beta_{c}} \frac{M\left(\beta, 0^{+}\right)}{\left(\frac{\beta-\beta_{c}}{\log \left(1 /\left(\beta-\beta_{c}\right)\right)}\right)^{1 / 2}}>0,
$$

concluding the proof for $\tau=5$.

Proof for exponent $\boldsymbol{\delta}$. We continue with the analysis for $\boldsymbol{\delta}$. Setting $\beta=\beta_{c}$ in (3.9), we obtain

$$
z^{*}\left(\beta_{c}, B\right) \leq\left(C_{1} \sqrt{\mathbb{E}[W]}\right)^{-\frac{1}{\delta}} B^{1 / \delta} .
$$

Using this inequality in (3.33) with $\beta=\beta_{c}$, we obtain

$$
M\left(\beta_{c}, B\right) \leq B+\frac{\mathbb{E}[W] C_{1}^{-\frac{1}{\delta}}}{\left(\sqrt{\mathbb{E}\left[W^{2}\right]}\right)^{1+\frac{1}{\delta}}} B^{1 / \delta},
$$

which proves that

$$
\limsup _{B \searrow 0} \frac{M\left(\beta_{c}, B\right)}{B^{1 / \delta}}<\infty
$$

since $\boldsymbol{\delta}>1$. Inequality (3.16) with $\beta=\beta_{c}$ gives

$$
z^{*}\left(\beta_{c}, B\right) \geq c_{1}^{-1 / \delta}\left(\frac{1}{\sqrt{\mathbb{E}\left[W^{2}\right]}}-e_{1}\left(\beta_{c}, B\right)\right)^{1 / \delta} B^{1 / \delta} .
$$

This estimate, along with (3.50), will be used in the lower bound of the magnetization at $\beta=\beta_{c}$ obtained by $\tanh x \geq x-x^{2}$ :

$$
M\left(\beta_{c}, B\right) \geq B+\frac{\mathbb{E}[W]}{\sqrt{\mathbb{E}\left[W^{2}\right]}}(1-2 B) z^{*}\left(\beta_{c}, B\right)-z^{*}\left(\beta_{c}, B\right)^{2}-B^{2},
$$

giving, for $B>0$ small,

$$
\begin{aligned}
M\left(\beta_{c}, B\right) & \geq B+\frac{\mathbb{E}[W]}{\sqrt{\mathbb{E}\left[W^{2}\right]}}(1-2 B) c_{1}^{-1 / \delta}\left(\frac{1}{\sqrt{\mathbb{E}\left[W^{2}\right]}}-e_{1}\left(\beta_{c}, B\right)\right)^{1 / \delta} B^{1 / \boldsymbol{\delta}} \\
& -\left(C_{1} \sqrt{\mathbb{E}[W]}\right)^{-2 / \delta} B^{2 / \delta}-B^{2} .
\end{aligned}
$$

Recalling that $\lim _{B \searrow 0} e_{1}\left(\beta_{c}, B\right)=0$ and $\boldsymbol{\delta}>1$, the previous bound gives

$$
\liminf _{B \searrow 0} \frac{M\left(\beta_{c}, B\right)}{B^{1 / \delta}} \geq \sqrt{\mathbb{E}[W]}\left(c_{1} \sqrt{\mathbb{E}\left[W^{2}\right]}\right)^{-1 / \delta}>0,
$$

which concludes the proof for $\boldsymbol{\delta}$ in the cases $\tau \in(3,5)$ and $\mathbb{E}\left[W^{2}\right]<\infty$. The analysis for $\tau=5$ can be performed in a similar way as for $\boldsymbol{\beta}$.

Proof of Corollary 2.14. The proof can be simply adapted as in [11, Corollary 2.9].

\footnotetext{
${ }^{2}$ The proof of the inequality $z_{0}^{*} \geq C\left(\beta-\beta_{c}\right)^{1 /(2-\varepsilon)}$, for $0<\varepsilon<2$ can be obtained starting from the rightmost inequality of (3.48) combined with the fact that $\log 1 / x \leq A_{\varepsilon} x^{-\varepsilon}$ for all $x \in(0,1)$ and any $\varepsilon>0$.
} 


\subsection{Susceptibility: critical exponents $\gamma$ and $\gamma^{\prime}$}

We now analyze the susceptibility and compute the critical exponents $\gamma$ and $\gamma^{\prime}$. We start by computing the former under more general conditions than those of Theorem 2.10 .

Theorem 3.6 (Value of $\gamma$ ). For $\mathbb{E}\left[W^{2}\right]<\infty$,

$$
\lim _{\beta \nearrow \beta_{c}} \chi\left(\beta, 0^{+}\right)\left(\beta_{c}-\beta\right)=\frac{\mathbb{E}[W]^{2}}{\mathbb{E}\left[W^{2}\right]} \tanh \left(\beta_{c}\right),
$$

so that $\gamma=1$.

Proof. From Theorem 2.8 it follows that in the one-phase region, i.e., for $\beta<\beta_{c}$ or $B \neq 0$,

$$
\chi(\beta, B)=\frac{\partial}{\partial B} M(\beta, B)=\mathbb{E}\left[\left(1+\alpha(\beta) W \frac{\partial z^{*}}{\partial B}\right)\left(1-\tanh ^{2}\left(\alpha(\beta) W z^{*}+B\right)\right)\right] .
$$

We can also compute the derivative of $z^{*}$ by taking the derivative of (2.23):

$$
\frac{\partial z^{*}}{\partial B}=\mathbb{E}\left[\left(\alpha(\beta) W+\alpha(\beta)^{2} W^{2} \frac{\partial z^{*}}{\partial B}\right)\left(1-\tanh ^{2}\left(\alpha(\beta) W z^{*}+B\right)\right)\right] .
$$

If we take the limit $B \searrow 0$ for $\beta<\beta_{c}$, then the $\tanh ^{2}(\cdot)$ term vanishes, since by definition of $\beta_{c}$ it holds that $z_{0}^{*} \equiv \lim _{B \backslash 0} z^{*}=0$. Hence, if we write

$$
\frac{\partial z_{0}^{*}}{\partial B}=\lim _{B \searrow 0} \frac{\partial}{\partial B} z^{*}(\beta, B)
$$

then (3.59) simplifies to

$$
\frac{\partial z_{0}^{*}}{\partial B}=\sqrt{\mathbb{E}[W] \sinh (\beta)}+\sinh (\beta) \nu \frac{\partial z_{0}^{*}}{\partial B} .
$$

Solving for $\frac{\partial z_{0}^{*}}{\partial B}$ gives

$$
\frac{\partial z_{0}^{*}}{\partial B}=\frac{\sqrt{\mathbb{E}[W] \sinh (\beta)}}{1-\sinh (\beta) \nu}
$$

Also taking the limit $B \searrow 0$ in (3.58) and using the above gives

$$
\chi\left(\beta, 0^{+}\right)=1+\frac{\mathbb{E}[W] \sinh (\beta)}{1-\sinh (\beta) \nu} .
$$

From a Taylor expansion around $\beta_{c}$, we get that

$$
\sinh \left(\beta_{c}\right)-\cosh \left(\beta_{c}\right)\left(\beta_{c}-\beta\right) \leq \sinh (\beta) \leq \sinh \left(\beta_{c}\right)-\cosh (\beta)\left(\beta_{c}-\beta\right),
$$

so that

$$
1+\frac{\mathbb{E}[W]^{2} \sinh (\beta)}{\mathbb{E}\left[W^{2}\right] \cosh \left(\beta_{c}\right)\left(\beta_{c}-\beta\right)} \leq \chi\left(\beta, 0^{+}\right) \leq 1+\frac{\mathbb{E}[W]^{2} \sinh (\beta)}{\mathbb{E}\left[W^{2}\right] \cosh (\beta)\left(\beta_{c}-\beta\right)},
$$

$\operatorname{since} \sinh \left(\beta_{c}\right) \nu=1$. Hence, (3.57) follows.

We now analyze $\gamma^{\prime}$ :

Theorem 3.7 (Value of $\gamma^{\prime}$ ). For $W$ satisfying Condition 2.4 with $\mathbb{E}\left[W^{4}\right]<\infty$ or with $\tau \in(3,5)$,

$$
\gamma^{\prime}=1
$$


Proof. We split the proof into the two cases that cover the hypotheses of the theorem.

(a) Proof under the assumption $\mathbb{E}\left[W^{4}\right]<\infty$. We are now in the regime where $\beta>\beta_{c}$, so that $z_{0}^{*}>0$. We start from (3.59), take the limit $B \searrow 0$ and linearize the hyperbolic tangent. In order to control this approximation, we define $g(x)=x^{2}-\tanh ^{2}(x)$ and remark that on the basis of our assumption on $W$, we have that $\mathbb{E}\left[\left(W^{2} \vee 1\right) g(W)\right]<\infty$. It will be useful also to factorize $g(x)=x^{4} k(x)$ with $k(x)=O(1)$ as $x \rightarrow 0$, so that we also have $\mathbb{E}\left[W^{6} k(W)\right]<\infty$. This gives

$$
\begin{aligned}
& \frac{\partial z_{0}^{*}}{\partial B}=\mathbb{E} {\left[\left(\alpha(\beta) W+\alpha(\beta)^{2} W^{2} \frac{\partial z_{0}^{*}}{\partial B}\right)\left(1-\tanh ^{2}\left(\alpha(\beta) W z_{0}^{*}\right)\right)\right] } \\
&=\sqrt{\mathbb{E}[W] \sinh (\beta)}-e_{0}+\frac{\partial z_{0}^{*}}{\partial B}\left(\sinh (\beta) \nu-\alpha(\beta)^{4} \mathbb{E}\left[W^{4}\right] z_{0}^{* 2}\right)+ \\
&+\mathbb{E}\left[\left(\frac{\partial z_{0}^{*}}{\partial B} \alpha(\beta)^{2} W^{2}+\alpha(\beta) W\right) g\left(\alpha(\beta) W z_{0}^{*}\right)\right],
\end{aligned}
$$

where

$$
e_{0}=\left(\frac{\sinh (\beta)}{\mathbb{E}[W]}\right)^{3 / 2} \mathbb{E}\left[W^{3}\right] z_{0}^{* 2}
$$

Solving (3.67) for $\frac{\partial z_{0}^{*}}{\partial B}$ gives

$$
\frac{\partial z_{0}^{*}}{\partial B}=\frac{\sqrt{\mathbb{E}[W] \sinh (\beta)}-e_{0}-\mathbb{E}\left[\alpha(\beta) W g\left(\alpha(\beta) W z_{0}^{*}\right)\right]}{1-\sinh (\beta) \nu+\alpha(\beta)^{4} \mathbb{E}\left[W^{4}\right] z_{0}^{* 2}-\mathbb{E}\left[\alpha(\beta)^{2} W^{2} g\left(\alpha(\beta) W z_{0}^{*}\right)\right]} .
$$

To analyze (3.69) we use the lower and upper bounds in Propositions 3.4 and 3.3 Taking the limit $B \searrow 0$ in (3.16) with $\boldsymbol{\delta}=3, c_{1}$ given in (3.20) and dividing by $z_{0}^{*}$, we obtain

$$
z_{0}^{* 2} \geq 3 \frac{\mathbb{E}[W]^{2}}{\mathbb{E}\left[W^{4}\right]} \frac{1}{\sinh ^{2}(\beta)}(\sinh (\beta) \nu-1) .
$$

Taking the same limit $B \searrow 0$ in (3.14) and dividing by $z_{0}^{*}$ we obtain also

$$
z_{0}^{* 2} \leq 6 \frac{\mathbb{E}[W]^{2}}{\mathbb{E}\left[W^{4} \mathbb{1}_{\left\{\alpha(\beta) W z^{*} \leq \operatorname{atanh} \frac{1}{8}\right\}}\right]} \frac{1}{\sinh ^{2}(\beta)}(\sinh (\beta) \nu-1) .
$$

By Taylor expansion,

$$
\sinh (\beta) \nu-1=\cosh \left(\beta_{c}\right) \nu\left(\beta-\beta_{c}\right)+O\left(\left(\beta-\beta_{c}\right)^{2}\right),
$$

we conclude, from (3.70), (3.71), and the fact that $\mathbb{E}\left[W^{4} \mathbb{1}_{\left\{\alpha(\beta) W z^{*} \leq \operatorname{atanh} \frac{1}{8}\right\}}\right] \rightarrow \mathbb{E}\left[W^{4}\right]$ as $\beta \rightarrow \beta_{c}$, that $z_{0}^{* 2}=$ $O\left(\beta-\beta_{c}\right)$. Using this, we can now evaluate the terms in numerator and denominator of (3.69) as $\beta \rightarrow \beta_{c}$. The first term in the numerator has a non vanishing finite limit, while $e_{0}=O\left(\beta-\beta_{c}\right)$. The third term (ignoring the irrelevant multiplicative factor $\alpha(\beta))$ is $\mathbb{E}\left[W g\left(\alpha(\beta) W z_{0}^{*}\right)\right]=\alpha(\beta)^{4} z_{0}^{* 4} \mathbb{E}\left[W^{5} k\left(\alpha(\beta) W z_{0}^{*}\right)\right]=$ $O\left(\left(\beta-\beta_{c}\right)\right)$. Indeed, since $k(x) \leq \frac{1}{x^{2}}$,

$$
\alpha(\beta)^{4} z_{0}^{* 4} \mathbb{E}\left[W^{5} k\left(\alpha(\beta) W z_{0}^{*}\right)\right] \leq \alpha(\beta)^{2} \mathbb{E}\left[W^{3}\right] z_{0}^{* 2}=O\left(\beta-\beta_{c}\right) .
$$

Let us now consider the denominator and define

$$
D(\beta):=1-\sinh (\beta) \nu+\alpha(\beta)^{4} \mathbb{E}\left[W^{4}\right] z_{0}^{* 2} .
$$

By (3.70), (3.71) and (3.72),

$$
2 \cosh \left(\beta_{c}\right) \nu\left(\beta-\beta_{c}\right)+O\left(\left(\beta-\beta_{c}\right)^{2}\right) \leq D(\beta) \leq(a(\beta)-1) \cosh \left(\beta_{c}\right) \nu\left(\beta-\beta_{c}\right)+O\left(\left(\beta-\beta_{c}\right)^{2}\right),
$$


where $a(\beta)$ is a function that converges to 6 as $\beta \rightarrow \beta_{c}$. Thus, from the previous display we obtain $D(\beta)=O\left(\beta-\beta_{c}\right)$. The fourth term in the denominator of (3.69), again discarding an irrelevant factor and arguing as before, is $E\left[W^{2} g\left(\alpha(\beta) W z_{0}^{*}\right)\right]=\alpha(\beta)^{4} z_{0}^{* 4} \mathbb{E}\left[W^{6} k\left(\alpha(\beta) W z_{0}^{*}\right)\right]=O\left(\left(\beta-\beta_{c}\right)^{2}\right)$. Therefore, summarizing our findings,

$$
\frac{\partial z_{0}^{*}}{\partial B}=O\left(\left(\beta-\beta_{c}\right)^{-1}\right) .
$$

From (3.58), the upper bound follows using (3.76):

$$
\chi(\beta, 0) \leq \mathbb{E}\left[\left(1+\alpha(\beta) W \frac{\partial z_{0}^{*}}{\partial B}\right)\right] \leq 1+\sqrt{\sinh (\beta) \mathbb{E}[W]} O\left(\left(\beta-\beta_{c}\right)^{-1}\right) .
$$

Similarly, for the lower bound we use that $1-\tanh ^{2}(x) \geq 1-x^{2}$ for every $x$, we obtain

$$
\begin{aligned}
\chi(\beta, 0) & \geq \mathbb{E}\left[\left(1+\alpha(\beta) W \frac{\partial z_{0}^{*}}{\partial B}\right)\left(1-\alpha(\beta)^{2} W^{2} z_{0}^{* 2}\right)\right] \\
& =1+\mathbb{E}\left[\alpha(\beta) W \frac{\partial z_{0}^{*}}{\partial B}\right]-\mathbb{E}\left[\alpha(\beta)^{2} W^{2} z_{0}^{* 2}\right]-\mathbb{E}\left[\alpha(\beta)^{3} W^{3} z_{0}^{* 2} \frac{\partial z_{0}^{*}}{\partial B}\right] \\
& =1+\sqrt{\sinh (\beta) \mathbb{E}[W]} O\left(\left(\beta-\beta_{c}\right)^{-1}\right)-\sinh (\beta) \nu O\left(\beta-\beta_{c}\right)-\alpha(\beta)^{3} \mathbb{E}\left[W^{3}\right] O(1),
\end{aligned}
$$

again starting from (3.58), using (3.76) and $z_{0}^{* 2}=O\left(\beta-\beta_{c}\right)$. From (3.77) and (3.78) we obtain

$$
0<\liminf _{\beta \backslash \beta_{c}} \chi\left(\beta, 0^{+}\right)\left(\beta-\beta_{c}\right) \leq \limsup _{\beta \searrow \beta_{c}} \chi\left(\beta, 0^{+}\right)\left(\beta-\beta_{c}\right)<\infty,
$$

proving the theorem in the case that $\mathbb{E}\left[W^{4}\right]<\infty$.

(b) Proof for $W$ satisfying Condition 2.4 (ii). Now we generalize the previous proof in order to encompass also the case of those $W$ whose distribution function $F(w)=1-\mathbb{P}(W>w)$ satisfies Condition 2.4(ii). We start by defining

$$
h_{\beta, B, z^{*}}(w)=\tanh \left(\alpha w z^{*}+B\right) \alpha w-\alpha^{2} w^{2} z^{*},
$$

where the dependence of $\alpha$ on $\beta$ has been dropped, and rewriting (2.23) as

$$
z^{*}=\mathbb{E}\left[h_{\beta, B, z^{*}}(W)\right]+\alpha^{2} z^{*} \mathbb{E}\left[W^{2}\right] .
$$

Using integration by parts,

$$
\begin{aligned}
\mathbb{E}\left[h_{\beta, B, z^{*}}(W)\right]= & \int_{0}^{+\infty} h_{\beta, B, z^{*}}(w) d F(w)=-\int_{0}^{+\infty} h_{\beta, B, z^{*}}(w) d(1-F(w)) \\
= & -\lim _{w \rightarrow+\infty}\left[h_{\beta, B, z^{*}}(w)(1-F(w))\right]+h_{\beta, B, z^{*}}(0)(1-F(0)) \\
& +\int_{0}^{+\infty} h_{\beta, B, z^{*}}^{\prime}(w)(1-F(w)) d w .
\end{aligned}
$$

The boundary terms in the previous display vanish and therefore

$$
\mathbb{E}\left[h_{\beta, B, z^{*}}(W)\right]=\int_{0}^{+\infty} h_{\beta, B, z^{*}}^{\prime}(w)(1-F(w)) d w .
$$

Taking into account that the power law of Condition 2.4(ii) holds for $w>w_{0}$, we write the previous integral as

$$
\mathbb{E}\left[h_{\beta, B, z^{*}}(W)\right]=\bar{G}\left(\beta, B, z^{*}\right)+\bar{J}\left(\beta, B, z^{*}\right),
$$


where

$$
\bar{G}\left(\beta, B, z^{*}\right):=\int_{0}^{w_{0}} h_{\beta, B, z^{*}}^{\prime}(w)(1-F(w)) d w, \quad \bar{J}\left(\beta, B, z^{*}\right):=\int_{w_{0}}^{+\infty} h_{\beta, B, z^{*}}^{\prime}(w)(1-F(w)) d w .
$$

Therefore, (3.81) can be rewritten as

$$
z^{*}=\bar{G}\left(\beta, B, z^{*}\right)+\bar{J}\left(\beta, B, z^{*}\right)+\alpha^{2} z^{*} \mathbb{E}\left[W^{2}\right] .
$$

Now we take the limit $B \searrow 0$ in the previous equation. Recalling that $z_{0}^{*}:=\lim _{B \searrow 0} z^{*}>0$, and since the following limits exist:

$$
\lim _{B \searrow 0} \bar{G}\left(\beta, B, z^{*}\right)=G\left(\beta, z_{0}^{*}\right), \quad \lim _{B \searrow 0} \bar{J}\left(\beta, B, z^{*}\right)=J\left(\beta, z_{0}^{*}\right)
$$

by bounded convergence, then we arrive to

$$
z_{0}^{*}=G\left(\beta, z_{0}^{*}\right)+J\left(\beta, z_{0}^{*}\right)+\alpha^{2} z_{0}^{*} \mathbb{E}\left[W^{2}\right] .
$$

In the next step we bound $J\left(\beta, z_{0}^{*}\right)$. From the definition of $\bar{J}\left(\beta, B, z^{*}\right)$ in (3.85), and Condition $2.4($ (ii),

$$
c_{W} \int_{w_{0}}^{+\infty} h_{\beta, B, z^{*}}^{\prime}(w) w^{-(\tau-1)} d w \leq \bar{J}\left(\beta, B, z^{*}\right) \leq C_{W} \int_{w_{0}}^{+\infty} h_{\beta, B, z^{*}}^{\prime}(w) w^{-(\tau-1)} d w .
$$

Applying the change of variable $y=\alpha z^{*} w$ leads to

$$
\int_{w_{0}}^{+\infty} h_{\beta, B, z^{*}}^{\prime}(w) w^{-(\tau-1)} d w=\alpha^{\tau-1} z^{* \tau-2} \int_{\alpha w_{0} z^{*}}^{+\infty}\left[\tanh (y+B)-y \tanh ^{2}(y+B)-y\right] y^{-(\tau-1)} d y .
$$

Therefore, denoting

$$
\bar{I}\left(\beta, B, z^{*}\right):=\int_{\alpha w_{0} z^{*}}^{+\infty}\left[\tanh (y+B)-y \tanh ^{2}(y+B)-y\right] y^{-(\tau-1)} d y,
$$

we can rewrite (3.89) as follows:

$$
c_{W} \alpha^{\tau-1} z^{* \tau-2} \bar{I}\left(\beta, B, z^{*}\right) \leq \bar{J}\left(\beta, B, z^{*}\right) \leq C_{W} \alpha^{\tau-1} z^{* \tau-2} \bar{I}\left(\beta, B, z^{*}\right) .
$$

Since, again by bounded convergence,

$$
\lim _{B \searrow 0} \bar{I}\left(\beta, B, z^{*}\right)=\int_{\alpha w_{0} z_{0}^{*}}^{+\infty}\left[\tanh (y)-y \tanh ^{2}(y)-y\right] y^{-(\tau-1)} d y=: I\left(\beta, z_{0}^{*}\right),
$$

we obtain from (3.89) that

$$
c_{W} \alpha^{\tau-1} z_{0}^{* \tau-2} I\left(\beta, z_{0}^{*}\right) \leq J\left(\beta, z_{0}^{*}\right) \leq C_{W} \alpha^{\tau-1} z_{0}^{* \tau-2} I\left(\beta, z_{0}^{*}\right) .
$$

On the other hand, $\operatorname{since} \tanh (y)-y \tanh ^{2}(y)-y<0$ for $y>0$, we also have

$$
\begin{aligned}
k(\tau) & :=\int_{1}^{+\infty}\left[y \tanh ^{2}(y)+y-\tanh (y)\right] y^{-(\tau-1)} d y \leq-I\left(\beta, z_{0}^{*}\right) \\
& \leq \int_{0}^{+\infty}\left[y \tanh ^{2}(y)+y-\tanh (y)\right] y^{-(\tau-1)} d y=: K(\tau) .
\end{aligned}
$$


Therefore, from (3.88), (3.94) and (3.95),

$$
z_{0}^{*} \geq G\left(\beta, z_{0}^{*}\right)-c_{W} \alpha^{\tau-1} z_{0}^{* \tau-2} K(\tau)+\alpha^{2} z_{0}^{*} \mathbb{E}\left[W^{2}\right]
$$

and

$$
z_{0}^{*} \leq G\left(\beta, z_{0}^{*}\right)-C_{W} \alpha^{\tau-1} z_{0}^{* \tau-2} k(\tau)+\alpha^{2} z_{0}^{*} \mathbb{E}\left[W^{2}\right] .
$$

The next step is to control the behaviour of $G\left(\beta, z_{0}^{*}\right)$ as $\beta \rightarrow \beta_{c}$. We start by showing that $G\left(\beta, z_{0}^{*}\right)$ is $O\left(z_{0}^{* 3}\right)$ as $\beta \rightarrow \beta_{c}$. From the definition of $G\left(\beta, z_{0}^{*}\right)$,

$$
G\left(\beta, z_{0}^{*}\right)=\int_{0}^{w_{0}}\left[-\alpha^{2} z_{0}^{*} w-\tanh ^{2}\left(\alpha z_{0}^{*} w\right) \alpha^{2} z_{0}^{*} w+\alpha \tanh \left(\alpha z_{0}^{*} w\right)\right](1-F(w)) d w .
$$

Since the function between the square brackets is negative for $y>0$ and decreasing, we have

$$
\begin{gathered}
0 \geq G\left(\beta, z_{0}^{*}\right) \geq\left[-\alpha^{2} w_{0} z_{0}^{*}-\alpha^{2} w_{0} z_{0}^{*} \tanh ^{2}\left(\alpha w_{0} z_{0}^{*}\right)+\alpha \tanh \left(\alpha w_{0} z_{0}^{*}\right)\right] \int_{0}^{w_{0}}(1-F(w)) d w \\
\geq\left[-\alpha^{2} w_{0} z_{0}^{*}-\alpha^{2} w_{0} z_{0}^{*} \tanh ^{2}\left(\alpha w_{0} z_{0}^{*}\right)+\alpha \tanh \left(\alpha w_{0} z_{0}^{*}\right)\right]=-\frac{4}{3} \alpha^{4} w^{3} z_{0}^{* 3}+O\left(z_{0}^{* 5}\right)
\end{gathered}
$$

where the last equality is obtained by Taylor expansion.

Thus, the previous inequality implies that $G\left(\beta, z_{0}^{*}\right)=O\left(z_{0}^{* 3}\right)$. Again, from (3.96) and (3.97) dividing by $z_{0}^{*}$,

$$
1-\alpha^{2} \mathbb{E}\left[W^{2}\right] \geq z_{0}^{* \tau-3}\left(G\left(\beta, z_{0}^{*}\right) z_{0}^{* 2-\tau}-c_{W} \alpha^{\tau-1} K(\tau)\right),
$$

and

$$
1-\alpha^{2} \mathbb{E}\left[W^{2}\right] \leq z_{0}^{* \tau-3}\left(G\left(\beta, z_{0}^{*}\right) z_{0}^{* 2-\tau}-C_{W} \alpha^{\tau-1} k(\tau)\right) .
$$

Since $G\left(\beta, z_{0}^{*}\right) z_{0}^{* 2-\tau}=O\left(z_{0}^{* 5-\tau}\right)$ and $\tau \in(3,5)$, the previous inequalities together with (3.72) imply that $z_{0}^{* \tau-3}=O\left(\beta-\beta_{c}\right)$ as $\beta \searrow \beta_{c}$.

Next, we consider the derivative of $z_{0}^{*}$. Again, taking the limit $B \searrow 0$ for $\beta>\beta_{c}$ of (3.59) we obtain

$$
\frac{\partial z_{0}^{*}}{\partial B}=\frac{\alpha \mathbb{E}[W]-\alpha \mathbb{E}\left[W \tanh ^{2}\left(\alpha W z_{0}^{*}\right)\right]}{1-\alpha^{2} \mathbb{E}\left[W^{2}\right]+\alpha^{2} \mathbb{E}\left[W^{2} \tanh ^{2}\left(\alpha W z_{0}^{*}\right)\right]} .
$$

Since the numerator has a finite positive limit as $\beta \searrow \beta_{c}$ (in particular, the second term is vanishing), we will focus on the denominator

$$
D_{2}(\beta):=1-\alpha^{2} \mathbb{E}\left[W^{2}\right]+\alpha^{2} \mathbb{E}\left[W^{2} \tanh ^{2}\left(\alpha W z_{0}^{*}\right)\right] .
$$

We start by decomposing the average

$$
\mathbb{E}\left[W^{2} \tanh ^{2}\left(\alpha W z_{0}^{*}\right)\right]=\mathbb{E}\left[W^{2} \tanh ^{2}\left(\alpha W z_{0}^{*}\right) \mathbb{1}_{\left\{W \leq w_{0}\right\}}\right]+\mathbb{E}\left[W^{2} \tanh ^{2}\left(\alpha W z_{0}^{*}\right) \mathbb{1}_{\left\{W>w_{0}\right\}}\right],
$$

and analyze the two terms separately. The first one can be bounded as follows

$$
0 \leq \mathbb{E}\left[W^{2} \tanh ^{2}\left(\alpha W z_{0}^{*}\right) \mathbb{1}_{\left\{W \leq w_{0}\right\}}\right] \leq \alpha^{2} w_{0}^{4} z_{0}^{* 2}
$$

showing that

$$
\mathbb{E}\left[W^{2} \tanh ^{2}\left(\alpha W z_{0}^{*}\right) \mathbb{1}_{\left\{W \leq w_{0}\right\}}\right]=O\left(z_{0}^{* 2}\right)=O\left(\left(\beta-\beta_{c}\right)^{\frac{2}{\tau-3}}\right),
$$


with the exponent satisfying $2 /(\tau-3)>1$ since $\tau \in(3,5)$. The second term can be treated with the integration by parts formula

$$
\begin{aligned}
& \mathbb{E}\left[W^{2} \tanh ^{2}\left(\alpha W z_{0}^{*}\right) \mathbb{1}_{\left\{W>w_{0}\right\}}\right]=-\lim _{w \rightarrow+\infty}\left[w^{2} \tanh ^{2}\left(\alpha w z_{0}^{*}\right)(1-F(w))\right] \\
& +w_{0}^{2} \tanh ^{2}\left(\alpha w_{0} z_{0}^{*}\right)\left(1-F\left(w_{0}\right)\right)+\int_{w_{0}}^{+\infty} \frac{\partial}{\partial w}\left[w^{2} \tanh ^{2}\left(\alpha w z_{0}^{*}\right)\right](1-F(w)) d w .
\end{aligned}
$$

Since $\tau>3$, from Condition 2.4 we conclude that the limit in the previous display vanishes. It is also simple to see that

$$
w_{0}^{2} \tanh ^{2}\left(\alpha w_{0} z_{0}^{*}\right)\left(1-F\left(w_{0}\right)\right)=O\left(z_{0}^{* 2}\right)=O\left(\left(\beta-\beta_{c}\right)^{\frac{2}{\tau-3}}\right) .
$$

From (3.103) and using (3.104), (3.106), (3.107), (3.108), we can write

$$
D_{2}(\beta)=\bar{D}_{2}(\beta)+O\left(\left(\beta-\beta_{c}\right)^{\frac{2}{\tau-3}}\right),
$$

with

$$
\bar{D}_{2}(\beta):=\left(1-\alpha^{2} \mathbb{E}\left[W^{2}\right]\right)+\alpha^{2} \int_{w_{0}}^{+\infty} \frac{\partial}{\partial w}\left[w^{2} \tanh ^{2}\left(\alpha w z_{0}^{*}\right)\right](1-F(w)) d w .
$$

The second term in the r.h.s. of (3.109) is $O\left(\left(\beta-\beta_{c}\right)^{s}\right)$ with $s>1$, therefore we can forget it since the first term of $\bar{D}_{2}(\beta)$ is $O\left(\beta-\beta_{c}\right)$. Now we focus on the second term of $\bar{D}_{2}(\beta)$.

By using (2.7) and applying the change of variable $y=\alpha z^{*} w$, we can bound the integral in the last display as

$$
\int_{w_{0}}^{+\infty} \frac{\partial}{\partial w}\left[w^{2} \tanh ^{2}\left(\alpha w z_{0}^{*}\right)\right](1-F(w)) d w \leq C_{W} \alpha^{\tau-3} z_{0}^{* \tau-3} M(\tau)
$$

where

$$
M(\tau):=\int_{0}^{+\infty}\left[2 y \tanh ^{2}(y)+2 y^{2} \tanh (y)\left(1-\tanh ^{2}(y)\right)\right] y^{-(\tau-1)} d y
$$

and the bound in (3.111) is obtained thanks to the positivity of the integrand. The convergence of the integral is ensured by the fact that this function is $O\left(y^{4-\tau}\right)$ close to $y=0$ with $1>4-\tau>-1$ and is $O\left(y^{-\tau+2}\right)$ as $y \rightarrow \infty$ with $-\tau+2<-1$. In a similar fashion, we can also obtain

$$
\int_{w_{0}}^{+\infty} \frac{\partial}{\partial w}\left[w^{2} \tanh ^{2}\left(\alpha w z_{0}^{*}\right)\right](1-F(w)) d w \geq c_{W} \alpha^{\tau-3} z_{0}^{* \tau-3} m(\tau)
$$

with

$$
m(\tau):=\int_{\varepsilon}^{+\infty}\left[2 y \tanh ^{2}(y)+2 y^{2} \tanh (y)\left(1-\tanh ^{2}(y)\right)\right] y^{-(\tau-1)} d y,
$$

for $\beta$ sufficiently close to $\beta_{c}$. At this stage $\varepsilon>0$ is an arbitrary fixed quantity that will be chosen later (but independently of $\beta$ ). By (3.96) and (3.97),

$$
\frac{G\left(\beta, z_{0}^{*}\right) z_{0}^{*-1}-\left(1-\alpha^{2} \mathbb{E}\left[W^{2}\right]\right)}{c_{W} \alpha^{\tau-1} K(\tau)} \leq z_{0}^{* \tau-3} \leq \frac{G\left(\beta, z_{0}^{*}\right) z_{0}^{*-1}-\left(1-\alpha^{2} \mathbb{E}\left[W^{2}\right]\right)}{C_{W} \alpha^{\tau-1} k(\tau)},
$$

which, substituted in (3.111) and (3.113), gives

$$
\begin{aligned}
G\left(\beta, z_{0}^{*}\right) z_{0}^{*-1} \frac{m(\tau)}{K(\tau)}-\left(1-\alpha^{2} \mathbb{E}\left[W^{2}\right]\right) \frac{m(\tau)}{K(\tau)} & \leq \alpha^{2} \int_{w_{0}}^{+\infty} \frac{\partial}{\partial w}\left[w^{2} \tanh ^{2}\left(\alpha w z_{0}^{*}\right)\right](1-F(w)) d w \\
& \leq G\left(\beta, z_{0}^{*}\right) z_{0}^{*-1} \frac{M(\tau)}{k(\tau)}-\left(1-\alpha^{2} \mathbb{E}\left[W^{2}\right]\right) \frac{M(\tau)}{k(\tau)}
\end{aligned}
$$


By definition of $\bar{D}_{2}(\beta)$,

$G\left(\beta, z_{0}^{*}\right) z_{0}^{*-1} \frac{m(\tau)}{K(\tau)}+\left(1-\alpha^{2} \mathbb{E}\left[W^{2}\right]\right)\left(1-\frac{m(\tau)}{K(\tau)}\right) \leq \bar{D}_{2}(\beta) \leq G\left(\beta, z_{0}^{*}\right) z_{0}^{*-1} \frac{M(\tau)}{k(\tau)}+\left(1-\alpha^{2} \mathbb{E}\left[W^{2}\right]\right)\left(1-\frac{M(\tau)}{k(\tau)}\right)$

In the last step of the proof, we show that $\frac{m(\tau)}{K(\tau)}>1$. This can be done by properly choosing the arbitrary quantity $\varepsilon$ in (3.114). We will prove the first inequality, the second one can be obtained in the same way. Starting from (3.95) and (3.114), we introduce the functions $K_{b}(\tau)$ and $m_{a}(\tau)$ for $a \geq 0, b \geq 0$ as

$$
K_{b}(\tau):=\int_{b}^{+\infty} \frac{d}{d y}\left[y^{2}-y \tanh (y)\right] y^{-(\tau-1)} d y, \quad m_{a}(\tau):=\int_{a}^{+\infty} \frac{d}{d y}\left[y^{2} \tanh ^{2}(y)\right] y^{-(\tau-1)} d y
$$

which coincide with $K(\tau)$ and $m(\tau)$ for $b=0$ and $a=\varepsilon$, respectively. By applying the integration by parts formula the two functions can be written as

$$
\begin{aligned}
& K_{b}(\tau)=-b^{3-\tau}+b^{2-\tau} \tanh (b)+(\tau-1) \int_{b}^{+\infty}\left(y^{2}-y \tanh (y)\right) y^{-\tau} d y \\
& m_{a}(\tau)=-a \tanh (a)+(\tau-1) \int_{a}^{+\infty} y^{2} \tanh ^{2}(y) y^{-\tau} d y
\end{aligned}
$$

Since

$$
\lim _{\substack{a \rightarrow 0^{+} \\ b \rightarrow 0^{+}}} \frac{m_{a}(\tau)}{K_{b}(\tau)}=\frac{m_{0}(\tau)}{K(\tau)}=\frac{\int_{0}^{+\infty} y^{2} \tanh ^{2}(y) y^{-\tau} d y}{\int_{0}^{+\infty}\left[y^{2}-y \tanh (y)\right] y^{-\tau} d y}>1
$$

where the inequality can be proved by observing that $y^{2} \tanh ^{2}(y)>y^{2}-y \tanh (y)$ for all $y>0$, then for any $\varepsilon>0$ sufficiently small,

$$
\frac{m(\tau)}{K(\tau)}>1
$$

Since $G\left(\beta, z_{0}^{*}\right) z_{0}^{*-1}=O\left(z_{0}^{* 2}\right)=O\left(\left(\beta-\beta_{c}\right)^{s}\right)$ with $s=\frac{2}{\tau-3}>1$ and $\left(1-\alpha^{2} \mathbb{E}\left[W^{2}\right]\right)=O\left(\beta-\beta_{c}\right)$, with $1-\alpha^{2} \mathbb{E}\left[W^{2}\right]<0$ for $\beta>\beta_{c}$ and close to $\beta_{c}$ (see (3.72) $)$, we conclude that $0<\bar{D}_{2}(\beta)=O\left(\beta-\beta_{c}\right)$, for the same values of $\beta$. This proves that

$$
0<\frac{\partial z_{0}^{*}}{\partial B}=O\left(\left(\beta-\beta_{c}\right)^{-1}\right)
$$

The previous equation together with $z_{0}^{* \tau-3}=O\left(\beta-\beta_{c}\right)$ allows us to conclude the proof along the same lines of the case with $\mathbb{E}\left[W^{4}\right]<\infty$. Indeed, the upper bound (3.77) is still valid in the present case, since only the first moment of $W$ is involved. For the lower bound we argue as follows. Since, $1-\tanh ^{2}(x)>$ $1-\tanh (x)>1-x$ for $x>0$, we have

$$
\begin{aligned}
\chi(\beta, 0) & \geq \mathbb{E}\left[\left(1+\alpha(\beta) W \frac{\partial z_{0}^{*}}{\partial B}\right)\left(1-\alpha(\beta) W z_{0}^{*}\right)\right] \\
& =1-\sqrt{\sinh (\beta) \mathbb{E}[W]} z_{0}^{*}+\sqrt{\sinh (\beta) \mathbb{E}[W]} \frac{\partial z_{0}^{*}}{\partial B}-\sinh (\beta) \nu z_{0}^{*} \frac{\partial z_{0}^{*}}{\partial B} \\
& =1-\sqrt{\sinh (\beta) \mathbb{E}[W]} O\left(\left(\beta-\beta_{c}\right)^{1 /(\tau-3)}\right)+\sqrt{\sinh (\beta) \mathbb{E}[W]} O\left(\left(\beta-\beta_{c}\right)^{-1}\right) \\
& -\sinh (\beta) \nu O\left(\left(\beta-\beta_{c}\right)^{1 /(\tau-3)}\right) O\left(\left(\beta-\beta_{c}\right)^{-1}\right) .
\end{aligned}
$$

The inequalities (3.77) and (3.123) imply (3.79) concluding the proof of the theorem. 


\section{Non-classical limit theorems at criticality: proof of Theorem 2.15}

In this section we prove Theorem 2.15. For this, we follow the strategy of the proof for the Curie-Weiss model (see e.g. [14, Theorem V.9.5]). It suffices to prove that for any real number $r$

$$
\lim _{N \rightarrow \infty} P_{N}\left(\exp \left(r \frac{S_{N}}{N^{\boldsymbol{\delta} /(\boldsymbol{\delta}+1)}}\right)\right)=\frac{\int_{-\infty}^{\infty} \exp (r z-f(z)) \mathrm{d} z}{\int_{-\infty}^{\infty} \exp (-f(z)) \mathrm{d} z}
$$

As observed in [18], the measure $P_{N}$ is approximately equal to the inhomogeneous Curie-Weiss measure

$$
\widetilde{P}_{N}(g)=\frac{1}{\widetilde{Z}_{N}} \sum_{\sigma \in \Omega_{N}} g(\sigma) \mathrm{e}^{\frac{1}{2} \sinh \beta \sum_{i, j \in[N]} \frac{w_{i} w_{j}}{\ell_{N}} \sigma_{i} \sigma_{j}}=\frac{1}{\widetilde{Z}_{N}} \sum_{\sigma \in \Omega_{N}} g(\sigma) \mathrm{e}^{\frac{1}{2} \frac{\sinh \beta}{\ell_{N}}\left(\sum_{i \in[N]} w_{i} \sigma_{i}\right)^{2}},
$$

where $g(\sigma)$ is any bounded function defined in $\Omega_{N}$ and $\widetilde{Z}_{N}$ is the associated normalization factor, i.e.,

$$
\widetilde{Z}_{N}=\sum_{\sigma \in \Omega_{N}} \mathrm{e}^{\frac{1}{2} \frac{\sinh \beta}{\ell_{N}}\left(\sum_{i \in[N]} w_{i} \sigma_{i}\right)^{2}}
$$

We first prove the theorem for this measure $\widetilde{P}_{N}$, which is the rank-1 inhomogeneous Curie-Weiss model with $\beta$ replaced with $\sinh (\beta)$.

For this, we use the Hubbard-Stratonovich identity to rewrite $\widetilde{P}_{N}\left(\exp \left(r \frac{S_{N}}{N^{\lambda}}\right)\right)$ as a fraction of two integrals of an exponential function in Lemma 4.1 in Section 4.1. We next split the analysis into the cases $\mathbb{E}\left[W^{4}\right]<\infty$ and $\tau \in(3,5)$ in Sections 4.2 and 4.3, respectively. For both these cases we analyze the exponents in the integrals and use Taylor expansions to show that they converge in Lemmas 4.2 and 4.4 . respectively. We then use dominated convergence to show that the integrals also converge in Lemmas 4.3 and 4.5, respectively. The tail behavior of $f(x)$ for $\tau \in(3,5)$ is analyzed in Lemma 4.6. Combining these results we conclude the proof of Theorem 2.15 in Section 4.4 we first prove the theorem for $\widetilde{P}_{N}$ and then we show that the theorem also holds for $P_{N}$ in Lemma 4.7. Finally, in Section 4.5, we discuss how to adapt the proof to obtain the results on the scaling window.

\subsection{Rewrite of the moment generating function}

To ease the notation we first rescale $S_{N}$ by $N^{\lambda}$ and later set $\lambda=\boldsymbol{\delta} /(\boldsymbol{\delta}+1)$. We rewrite $\widetilde{P}_{N}\left(\exp \left(r \frac{S_{N}}{N^{\lambda}}\right)\right)$ in the following lemma:

Lemma 4.1 (Moment generating function of $S_{N} / N^{\lambda}$ ). For $B=0$,

$$
\widetilde{P}_{N}\left(\exp \left(r \frac{S_{N}}{N^{\lambda}}\right)\right)=\frac{\int_{-\infty}^{\infty} \mathrm{e}^{-N G_{N}(z ; r)} \mathrm{d} z}{\int_{-\infty}^{\infty} \mathrm{e}^{-N G_{N}(z ; 0)} \mathrm{d} z}
$$

where

$$
G_{N}(z ; r)=\frac{1}{2} z^{2}-\mathbb{E}\left[\log \cosh \left(\alpha_{N}(\beta) W_{N} z+\frac{r}{N^{\lambda}}\right)\right]
$$

with

$$
\alpha_{N}(\beta)=\sqrt{\frac{\sinh \beta}{\mathbb{E}\left[W_{N}\right]}}
$$


Proof. We use the Hubbard-Stratonovich identity, i.e., we write $\mathrm{e}^{t^{2} / 2}=\mathbb{E}\left[\mathrm{e}^{t Z}\right]$, with $Z$ standard Gaussian, to obtain

$$
\begin{aligned}
\widetilde{Z}_{N} \widetilde{P}_{N}\left(\exp \left(r \frac{S_{N}}{N^{\lambda}}\right)\right) & =\sum_{\sigma \in \Omega_{N}} \mathrm{e}^{\frac{r}{N^{\lambda}} \sum_{i \in[N]} \sigma_{i}} \mathrm{e}^{\frac{1}{2} \frac{\sinh \beta}{\ell_{N}}\left(\sum_{i \in[N]} w_{i} \sigma_{i}\right)^{2}} \\
& =\sum_{\sigma \in \Omega_{N}} \mathrm{e}^{\frac{r}{N^{\lambda}} \sum_{i \in[N]} \sigma_{i}} \mathbb{E}\left[\mathrm{e}^{\sqrt{\frac{\sinh \beta}{\ell_{N}}}\left(\sum_{i \in[N]} w_{i} \sigma_{i}\right) Z}\right] \\
& =2^{N} \mathbb{E}\left[\prod_{i \in[N]} \cosh \left(\sqrt{\frac{\sinh \beta}{\ell_{N}}} w_{i} Z+\frac{r}{N^{\lambda}}\right)\right]=2^{N} \mathbb{E}\left[\mathrm{e}^{\sum_{i \in[N]} \log \cosh \left(\sqrt{\frac{\sinh \beta}{\ell_{N}}} w_{i} Z+\frac{r}{N^{\lambda}}\right)}\right] .
\end{aligned}
$$

We rewrite the sum in the exponential, using the fact that $W_{N}=w_{U_{N}}$, where $U_{N}$ is a uniformly chosen vertex in $[N]$, as

$$
\begin{aligned}
\widetilde{Z}_{N} \widetilde{P}_{N}\left(\exp \left(r \frac{S_{N}}{N^{\lambda}}\right)\right) & =2^{N} \mathbb{E}\left[\exp \left\{N \mathbb{E}\left[\log \cosh \left(\sqrt{\frac{\sinh \beta}{N \mathbb{E}\left[W_{N}\right]}} W_{N} Z+\frac{r}{N^{\lambda}}\right) \mid Z\right]\right\}\right] \\
& =\frac{2^{N}}{\sqrt{2 \pi}} \int_{-\infty}^{\infty} \mathrm{e}^{-z^{2} / 2} \exp \left\{N \mathbb{E}\left[\log \cosh \left(\alpha_{N}(\beta) W_{N} \frac{z}{\sqrt{N}}+\frac{r}{N^{\lambda}}\right)\right]\right\} \mathrm{d} z
\end{aligned}
$$

By substituting $z / \sqrt{N}$ for $z$, we get

$$
\begin{aligned}
\widetilde{Z}_{N} \widetilde{P}_{N}\left(\exp \left(r \frac{S_{N}}{N^{\lambda}}\right)\right) & =2^{N} \sqrt{\frac{N}{2 \pi}} \int_{-\infty}^{\infty} \mathrm{e}^{-N z^{2} / 2} \exp \left\{N \mathbb{E}\left[\log \cosh \left(\alpha_{N}(\beta) W_{N} z+\frac{r}{N^{\lambda}}\right)\right]\right\} \mathrm{d} z \\
& =2^{N} \sqrt{\frac{N}{2 \pi}} \int_{-\infty}^{\infty} \mathrm{e}^{-N G_{N}(z ; r)} \mathrm{d} z .
\end{aligned}
$$

In a similar way we can rewrite

$$
\widetilde{Z}_{N}=2^{N} \sqrt{\frac{N}{2 \pi}} \int_{-\infty}^{\infty} \mathrm{e}^{-N G_{N}(z ; 0)} \mathrm{d} z
$$

so that the lemma follows.

\subsection{Convergence for $\mathbb{E}\left[W^{4}\right]<\infty$}

We analyze the asymptotics of the function $G_{N}(z ; r)$ :

Lemma 4.2 (Asymptotics of $G_{N}$ for $\mathbb{E}\left[W^{4}\right]<\infty$ ). For $\beta=\beta_{c, N}, B=0$ and $\mathbb{E}\left[W^{4}\right]<\infty$,

$$
\lim _{N \rightarrow \infty} N G_{N}\left(z / N^{1 / 4} ; r\right)=-z r \sqrt{\frac{\mathbb{E}[W]}{\nu}}+\frac{1}{12} \frac{\mathbb{E}\left[W^{4}\right]}{\mathbb{E}\left[W^{2}\right]^{2}} z^{4}
$$

Proof. Taylor expanding $\log \cosh (x)$ about $x=0$ gives that

$$
\log \cosh (x)=\frac{x^{2}}{2}-\frac{1}{12} x^{4}+O\left(x^{6}\right) .
$$

We want to use this to analyze $N G_{N}\left(z / N^{1 / 4} ; r\right)$ and hence need to analyze the second, fourth and sixth moment of $\sqrt{\frac{\sinh \beta_{c, N}}{\mathbb{E}\left[W_{N}\right]}} W_{N} \frac{z}{N^{1 / 4}}+\frac{r}{N^{\lambda}}$. 
The second moment equals, using that $\lambda=\boldsymbol{\delta} /(\boldsymbol{\delta}+1)=3 / 4$,

$$
\begin{aligned}
\mathbb{E}\left[\left(\alpha_{N}\left(\beta_{c, N}\right) W_{N} \frac{z}{N^{1 / 4}}+\frac{r}{N^{\lambda}}\right)^{2}\right] & =\sinh \beta_{c, N} \nu_{N} \frac{z^{2}}{\sqrt{N}}+2 \sqrt{\sinh \beta_{c, N} \mathbb{E}\left[W_{N}\right]} \frac{z r}{N}+\frac{r^{2}}{N^{6 / 4}} \\
& =\frac{z^{2}}{\sqrt{N}}+2 \frac{z r}{N} \sqrt{\frac{\mathbb{E}\left[W_{N}\right]}{\nu_{N}}}+o(1 / N),
\end{aligned}
$$

where we have used that $\sinh \beta_{c, N}=1 / \nu_{N}$ in the second equality.

For the fourth moment we use that by assumption the first four moments of $W_{N}$ are $O(1)$. Hence, for all $r$,

$$
\begin{aligned}
\mathbb{E}\left[\left(\alpha_{N}\left(\beta_{c, N}\right) W_{N} \frac{z}{N^{1 / 4}}+\frac{r}{N^{\lambda}}\right)^{4}\right] & =\frac{\sinh ^{2} \beta_{c, N}}{\mathbb{E}\left[W_{N}\right]^{2}} \mathbb{E}\left[W_{N}^{4}\right] \frac{z^{4}}{N}+O\left(\frac{1}{N^{3 / 4+\lambda}}+\frac{1}{N^{2 / 4+2 \lambda}}+\frac{1}{N^{1 / 4+3 \lambda}}+\frac{1}{N^{4 \lambda}}\right) \\
& =\frac{\mathbb{E}\left[W_{N}^{4}\right]}{\mathbb{E}\left[W_{N}^{2}\right]^{2}} \frac{z^{4}}{N}+o(1 / N)
\end{aligned}
$$

For the sixth moment, we have to be a bit more careful since $\mathbb{E}\left[W^{6}\right]$ is potentially infinite. We can, however, use that

$$
\mathbb{E}\left[W_{N}^{6}\right]=\frac{1}{N} \sum_{i=1}^{N} w_{i}^{6} \leq\left(\max _{i=1}^{N} w_{i}\right)^{2} \frac{1}{N} \sum_{i=1}^{N} w_{i}^{4}=\left(\max _{i} w_{i}\right)^{2} \mathbb{E}\left[W_{N}^{4}\right]
$$

It can easily be seen that $\max _{i=1}^{N} w_{i}=o\left(N^{1 / 4}\right)$ when $W_{N} \stackrel{\mathcal{D}}{\longrightarrow} W$ and $\mathbb{E}\left[W_{N}^{4}\right] \rightarrow \mathbb{E}\left[W^{4}\right]<\infty$. Hence,

$$
\mathbb{E}\left[\left(\alpha_{N}\left(\beta_{c, N}\right) W_{N} \frac{z}{N^{1 / 4}}\right)^{6}\right]=\frac{\sinh ^{3} \beta_{c, N}}{\mathbb{E}\left[W_{N}\right]^{3}} \mathbb{E}\left[W_{N}^{6}\right] \frac{z^{6}}{N^{6 / 4}}=\frac{o\left(N^{1 / 2}\right) \mathbb{E}\left[W_{N}^{4}\right]}{\mathbb{E}\left[W_{N}^{2}\right]^{3}} \frac{z^{6}}{N^{6 / 4}}=o(1 / N) .
$$

In a similar way, it can be shown that

$$
\mathbb{E}\left[\left(\alpha_{N}\left(\beta_{c, N}\right) W_{N} \frac{z}{N^{1 / 4}}+\frac{r}{N^{\lambda}}\right)^{6}\right]=o(1 / N) .
$$

Putting everything together and using that the first four moments of $W_{N}$ converge by assumption,

$$
\begin{aligned}
\lim _{N \rightarrow \infty} N G_{N}\left(z / N^{1 / 4} ; r\right) & =\lim _{N \rightarrow \infty}\left(\frac{\sqrt{N}}{2} z^{2}-N \mathbb{E}\left[\log \cosh \left(\alpha_{N}\left(\beta_{c, N}\right) W_{N} \frac{z}{N^{1 / 4}}+\frac{r}{N^{\lambda}}\right)\right]\right) \\
& =-z r \sqrt{\frac{\mathbb{E}[W]}{\nu}}+\frac{1}{12} \frac{\mathbb{E}\left[W^{4}\right]}{\mathbb{E}\left[W^{2}\right]^{2}} z^{4} .
\end{aligned}
$$

From Lemma 4.2 it also follows that the integral converges as we show next:

Lemma 4.3 (Convergence of the integral for $\mathbb{E}\left[W^{4}\right]<\infty$ ). For $\beta=\beta_{c, N}, B=0$ and $\mathbb{E}\left[W^{4}\right]<\infty$,

$$
\lim _{N \rightarrow \infty} \int_{-\infty}^{\infty} \mathrm{e}^{-N G_{N}\left(z / N^{1 / 4} ; r\right)} \mathrm{d} z=\int_{-\infty}^{\infty} \mathrm{e}^{z r \frac{\mathbb{E}[W]}{\sqrt{\mathbb{E}\left[W^{2}\right]}}-\frac{1}{12} \frac{\mathbb{E}\left[W^{4}\right]}{\mathbb{E}\left[W^{2}\right]^{2}} z^{4}} \mathrm{~d} z
$$


Proof. We prove this lemma using dominated convergence. Hence, we need to find a lower bound on $N G_{N}\left(z / N^{1 / 4} ; r\right)$. We first rewrite this function by using that

$$
\mathbb{E}\left[\frac{1}{2} \alpha_{N}\left(\beta_{c, N}\right)^{2} W_{N}^{2}\left(\frac{z}{N^{1 / 4}}\right)^{2}\right]=\frac{1}{2}\left(\frac{z}{N^{1 / 4}}\right)^{2} .
$$

Hence,

$$
\begin{aligned}
G_{N}\left(z / N^{1 / 4} ; r\right)=\mathbb{E} & {\left[\frac{1}{2} \alpha_{N}\left(\beta_{c, N}\right)^{2} W_{N}^{2}\left(\frac{z}{N^{1 / 4}}\right)^{2}-\log \cosh \left(\alpha_{N}\left(\beta_{c, N}\right) W_{N} \frac{z}{N^{1 / 4}}+\frac{r}{N^{\lambda}}\right)\right] } \\
=\mathbb{E} & {\left[\frac{1}{2}\left(\alpha_{N}\left(\beta_{c, N}\right) W_{N} \frac{z}{N^{1 / 4}}\right)^{2}-\log \cosh \left(\alpha_{N}\left(\beta_{c, N}\right) W_{N} \frac{z}{N^{1 / 4}}\right)\right] } \\
& -\mathbb{E}\left[\log \cosh \left(\alpha_{N}\left(\beta_{c, N}\right) W_{N} \frac{z}{N^{1 / 4}}+\frac{r}{N^{\lambda}}\right)-\log \cosh \left(\alpha_{N}\left(\beta_{c, N}\right) W_{N} \frac{z}{N^{1 / 4}}\right)\right]
\end{aligned}
$$

Since

$$
\frac{\mathrm{d}^{2}}{\mathrm{~d} x^{2}}\left(\frac{1}{2} x^{2}-\log \cosh x\right)=1-\left(1-\tanh ^{2}(x)\right)=\tanh ^{2}(x) \geq 0,
$$

the function $\frac{1}{2} x^{2}-\log \cosh x$ is convex and we can use Jensen's inequality to bound

$$
\begin{aligned}
& \mathbb{E}\left[\frac{1}{2}\left(\alpha_{N}\left(\beta_{c, N}\right) W_{N} \frac{z}{N^{1 / 4}}\right)^{2}-\log \cosh \left(\alpha_{N}\left(\beta_{c, N}\right) W_{N} \frac{z}{N^{1 / 4}}\right)\right] \\
& \quad \geq \frac{1}{2}\left(\alpha_{N}\left(\beta_{c, N}\right) \mathbb{E}\left[W_{N}\right] \frac{z}{N^{1 / 4}}\right)^{2}-\log \cosh \left(\alpha_{N}\left(\beta_{c, N}\right) \mathbb{E}\left[W_{N}\right] \frac{z}{N^{1 / 4}}\right) \\
& \quad=\frac{1}{2}\left(\sqrt{\frac{\mathbb{E}\left[W_{N}\right]}{\nu_{N}}} \frac{z}{N^{1 / 4}}\right)^{2}-\log \cosh \left(\sqrt{\frac{\mathbb{E}\left[W_{N}\right]}{\nu_{N}}} \frac{z}{N^{1 / 4}}\right) .
\end{aligned}
$$

As observed in the proof of [14, Theorem V.9.5], there exist positive constants $A$ and $\varepsilon$ so that

$$
\frac{1}{2} x^{2}-\log \cosh x \geq d(x):= \begin{cases}\varepsilon x^{4}, & \text { for }|x| \leq A \\ \varepsilon x^{2}, & \text { for }|x|>A\end{cases}
$$

To bound the second term in (4.21), we can use the Taylor expansion

$$
\log \cosh (a+x)=\log \cosh (a)+\tanh (\xi) x,
$$

for some $\xi \in(a, a+x)$, and that $|\tanh (\xi)| \leq|\xi| \leq|a|+|x|$ to obtain

$$
\begin{aligned}
\mathbb{E}[\log \cosh & \left.\left(\alpha_{N}\left(\beta_{c, N}\right) W_{N} \frac{z}{N^{1 / 4}}+\frac{r}{N^{\lambda}}\right)-\log \cosh \left(\alpha_{N}\left(\beta_{c, N}\right) W_{N} \frac{z}{N^{1 / 4}}\right)\right] \\
& \leq \mathbb{E}\left[\left|\log \cosh \left(\alpha_{N}\left(\beta_{c, N}\right) W_{N} \frac{z}{N^{1 / 4}}+\frac{r}{N^{\lambda}}\right)-\log \cosh \left(\alpha_{N}\left(\beta_{c, N}\right) W_{N} \frac{z}{N^{1 / 4}}\right)\right|\right] \\
& \leq \mathbb{E}\left[\left(\left|\alpha_{N}\left(\beta_{c, N}\right) W_{N} \frac{z}{N^{1 / 4}}\right|+\frac{|r|}{N^{\lambda}}\right) \frac{|r|}{N^{\lambda}}\right]=\alpha_{N}\left(\beta_{c, N}\right) \mathbb{E}\left[W_{N}\right] \frac{|z r|}{N^{1 / 4+\lambda}}+\frac{r^{2}}{N^{2 \lambda}} \\
& =\sqrt{\frac{\mathbb{E}\left[W_{N}\right]}{\nu_{N}}} \frac{|z r|}{N}+\frac{r^{2}}{N^{3 / 2}} .
\end{aligned}
$$

Hence,

$$
\mathrm{e}^{-N G_{N}\left(z / N^{1 / 4} ; r\right)} \leq \exp \left\{\sqrt{\frac{\mathbb{E}\left[W_{N}\right]}{\nu_{N}}}|z r|+\frac{r^{2}}{N^{1 / 2}}-N d\left(\sqrt{\frac{\mathbb{E}\left[W_{N}\right]}{\nu_{N}}} \frac{z}{N^{1 / 4}}\right)\right\}
$$


which we use as the dominating function. Hence, we need to prove that the integral of this function over $z \in \mathbb{R}$ is uniformly bounded. We split the integral as

$$
\begin{aligned}
& \int_{-\infty}^{\infty} \exp \left\{\sqrt{\frac{\mathbb{E}\left[W_{N}\right]}{\nu_{N}}}|z r|+\frac{r^{2}}{N^{1 / 2}}-N d\left(\sqrt{\frac{\mathbb{E}\left[W_{N}\right]}{\nu_{N}}} \frac{z}{N^{1 / 4}}\right)\right\} \mathrm{d} z
\end{aligned}
$$

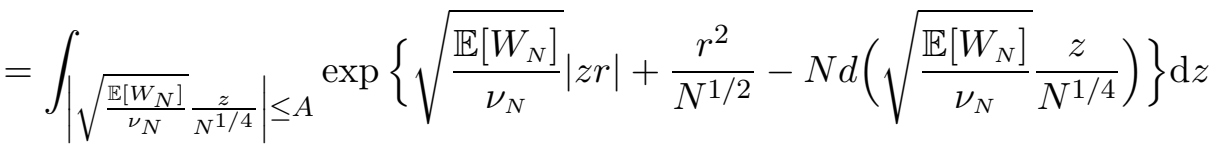

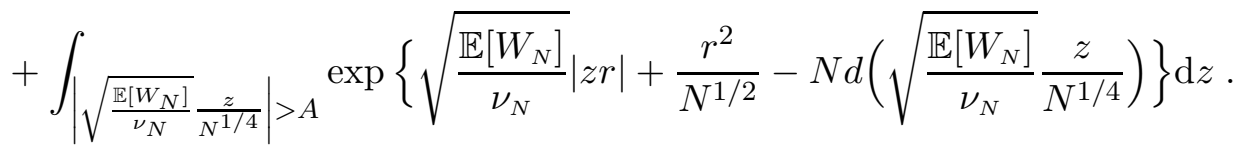

The first integral equals

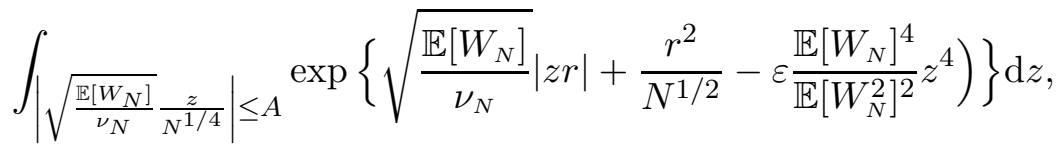

which clearly is uniformly bounded. The second integral equals

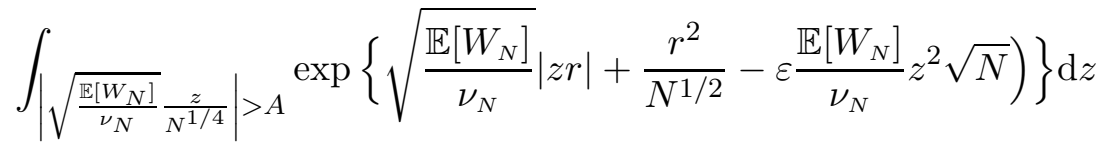

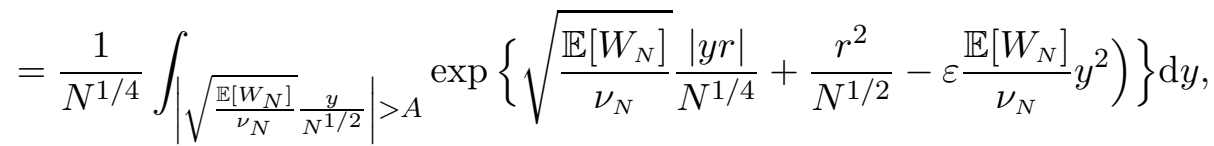

where we have substituted $y=z N^{1 / 4}$. This converges to zero for $N \rightarrow \infty$, because the integral is uniformly bounded.

Together with the pointwise convergence proved in Lemma 4.2, this proves Lemma 4.3 .

\subsection{Convergence for $\tau \in(3,5)$}

We next analyze $G_{N}(z ; r)$ for $\tau \in(3,5)$, assuming Condition 2.5 .

Lemma 4.4 (Asymptotics of $G_{N}$ for $\tau \in(3,5)$ ). Assume that Condition 2.5(ii) holds. For $\beta=\beta_{c, N}$, $B=0$ and $\tau \in(3,5)$,

$$
\lim _{N \rightarrow \infty} N G_{N}\left(z / N^{1 /(\tau-1)} ; r\right)=-z r \sqrt{\frac{\mathbb{E}[W]}{\nu}}+f\left(\sqrt{\left.\frac{\mathbb{E}[W]}{\nu} z\right),}\right.
$$

where $f(z)$ is defined in (2.37).

Proof. Define the function

$$
g(w, z)=\frac{1}{2}\left(\alpha_{N}\left(\beta_{c, N}\right) w z\right)^{2}-\log \cosh \left(\alpha_{N}\left(\beta_{c, N}\right) w z\right),
$$

so that we can rewrite, in a similar way as in (4.21),

$$
\begin{aligned}
& N G_{N}\left(z / N^{\frac{1}{\tau-1}} ; r\right)=N \mathbb{E}\left[g\left(W_{N}, z / N^{\frac{1}{\tau-1}}\right)\right] \\
& -N \mathbb{E}\left[\log \cosh \left(\frac{1}{\sqrt{\mathbb{E}\left[W_{N}^{2}\right]}} W_{N} \frac{z}{N^{\frac{1}{\tau-1}}}+\frac{r}{N^{\lambda}}\right)-\log \cosh \left(\frac{1}{\sqrt{\mathbb{E}\left[W_{N}^{2}\right]}} W_{N} \frac{z}{N^{\frac{1}{\tau-1}}}\right)\right] .
\end{aligned}
$$


By the definition of $W_{N}$, we can rewrite

$$
\mathbb{E}\left[g\left(W_{N}, z / N^{1 /(\tau-1)}\right)\right]=\frac{1}{N} \sum_{i=1}^{N} g\left(w_{i}, z / N^{1 /(\tau-1)}\right) .
$$

With the deterministic choice of the weights as in (2.8),

$$
\begin{aligned}
g\left(w_{i}, z / N^{1 /(\tau-1)}\right) & =\frac{1}{2}\left(\alpha_{N}\left(\beta_{c, N}\right) \frac{w_{i} z}{N^{1 /(\tau-1)}}\right)^{2}-\log \cosh \left(\alpha_{N}\left(\beta_{c, N}\right) \frac{w_{i} z}{N^{1 /(\tau-1)}}\right) \\
& =\frac{1}{2}\left(\frac{1}{\sqrt{\mathbb{E}\left[W_{N}^{2}\right]}} \frac{c_{w} z}{i^{1 /(\tau-1)}}\right)^{2}-\log \cosh \left(\frac{1}{\sqrt{\mathbb{E}\left[W_{N}^{2}\right]}} \frac{c_{w} z}{i^{1 /(\tau-1)}}\right) .
\end{aligned}
$$

From this it clearly follows that, for all $i \geq 1$,

$$
\lim _{N \rightarrow \infty} g\left(w_{i}, z / N^{1 /(\tau-1)}\right)=\frac{1}{2}\left(\frac{1}{\sqrt{\mathbb{E}\left[W^{2}\right]}} \frac{c_{w} z}{i^{1 /(\tau-1)}}\right)^{2}-\log \cosh \left(\frac{1}{\sqrt{\mathbb{E}\left[W^{2}\right]}} \frac{c_{w} z}{i^{1 /(\tau-1)}}\right) .
$$

It remains to show that also the sum converges, which we do using dominated convergence. For this, we use a Taylor expansion of $\log \cosh (x)$ about $x=0$ up to the fourth order

$$
\log \cosh (x)=\frac{x^{2}}{2}+\left(-2+2 \tanh ^{2}(\xi)+6 \tanh ^{2}(\xi)\left(1-\tanh ^{2}(\xi)\right)\right) \frac{x^{4}}{4 !} \geq \frac{x^{2}}{2}-\frac{x^{4}}{12},
$$

for some $\xi \in(0, x)$. Hence,

$$
g\left(w_{i}, z / N^{1 /(\tau-1)}\right) \leq \frac{1}{12}\left(\frac{1}{\sqrt{\mathbb{E}\left[W_{N}^{2}\right]}} \frac{c_{w} z}{i^{1 /(\tau-1)}}\right)^{4}=\frac{1}{12} \frac{1}{\mathbb{E}\left[W^{2}\right]^{2}+o(1)} \frac{\left(c_{w} z\right)^{4}}{i^{4 /(\tau-1)}} .
$$

Since $\tau \in(3,5)$, it holds that $4 /(\tau-1)>1$, so that

$$
\lim _{N \rightarrow \infty} \sum_{i=1}^{N} \frac{1}{12} \frac{1}{\mathbb{E}\left[W^{2}\right]^{2}+o(1)} \frac{\left(c_{w} z\right)^{4}}{i^{4 /(\tau-1)}}<\infty .
$$

We conclude that

$$
\begin{aligned}
\lim _{N \rightarrow \infty} \sum_{i=1}^{N} g\left(w_{i}, z / N^{1 /(\tau-1)}\right) & =\sum_{i=1}^{\infty}\left(\frac{1}{2}\left(\frac{1}{\sqrt{\mathbb{E}\left[W^{2}\right]}} \frac{c_{w} z}{i^{1 /(\tau-1)}}\right)^{2}-\log \cosh \left(\frac{1}{\sqrt{\mathbb{E}\left[W^{2}\right]}} \frac{c_{w} z}{i^{1 /(\tau-1)}}\right)\right) \\
& =f\left(\sqrt{\frac{\mathbb{E}[W]}{\nu} z}\right),
\end{aligned}
$$

where in the last equality we have used that $\mathbb{E}[W]=c_{w} \frac{\tau-1}{\tau-2}$. This is in turn a consequence of the following explicit computation giving an upper and lower bound on $\mathbb{E}\left[W_{N}\right]$ matching in the limit $N \rightarrow \infty$. An upper bound on the first moment is given by

$$
\begin{aligned}
\mathbb{E}\left[W_{N}\right] & =\frac{1}{N} \sum_{i=1}^{N} c_{w}\left(\frac{N}{i}\right)^{1 /(\tau-1)}=c_{w} N^{-\frac{\tau-2}{\tau-1}} \sum_{i=1}^{N} c_{w} i^{-1 /(\tau-1)} \leq c_{w} N^{-\frac{\tau-2}{\tau-1}}\left(1+\int_{1}^{N} i^{-1 /(\tau-1)} \mathrm{d} i\right) \\
& =c_{w} \frac{\tau-1}{\tau-2}-c_{w} \frac{1}{\tau-2} N^{-\frac{\tau-2}{\tau-1}}
\end{aligned}
$$


and a lower bound by

$$
\mathbb{E}\left[W_{N}\right] \geq c_{w} N^{-\frac{\tau-2}{\tau-1}} \int_{1}^{N} i^{-1 /(\tau-1)} \mathrm{d} i=c_{w} \frac{\tau-1}{\tau-2}-c_{w} \frac{\tau-1}{\tau-2} N^{-\frac{\tau-2}{\tau-1}} .
$$

From this it indeed follows that

$$
\mathbb{E}[W]=\lim _{N \rightarrow \infty} \mathbb{E}\left[W_{N}\right]=c_{w} \frac{\tau-1}{\tau-2} .
$$

To analyze the second term in (4.33), we can use the Taylor expansions

$$
\begin{aligned}
\log \cosh (a+x) & =\log \cosh (a)+\tanh (a) x+\left(1-\tanh ^{2}(\xi)\right) x^{2} \\
& =\log \cosh (a)+\left(a-\tanh \zeta\left(1-\tanh ^{2} \zeta\right) a^{2}\right) x+\left(1-\tanh ^{2}(\xi)\right) x^{2},
\end{aligned}
$$

for some $\xi \in(a, a+x)$ and $\zeta \in(0, a)$. This gives

$$
\begin{aligned}
N \mathbb{E}[\log \cosh & \left.\left(\frac{1}{\sqrt{\mathbb{E}\left[W_{N}^{2}\right]}} W_{N} \frac{z}{N^{\frac{1}{\tau-1}}}+\frac{r}{N^{\lambda}}\right)-\log \cosh \left(\frac{1}{\sqrt{\mathbb{E}\left[W_{N}^{2}\right]}} W_{N} \frac{z}{N^{\frac{1}{\tau-1}}}\right)\right] \\
& =N \sqrt{\frac{\mathbb{E}\left[W_{N}\right]}{\nu_{N}}} \frac{z}{N^{\frac{1}{\tau-1}}} \frac{r}{N^{\lambda}}-N \mathbb{E}\left[\tanh \zeta\left(1-\tanh ^{2} \zeta\right) W_{N}^{2}\right] \frac{1}{\mathbb{E}\left[W_{N}^{2}\right]} \frac{z^{2}}{N^{\frac{2}{\tau-1}}} \frac{r}{N^{\lambda}} \\
\quad & N \mathbb{E}\left[\left(1-\tanh ^{2}(\xi)\right)\right] \frac{r^{2}}{N^{2 \lambda}} \\
= & \sqrt{\frac{\mathbb{E}\left[W_{N}\right]}{\nu_{N}}} z r+o(1),
\end{aligned}
$$

where the last equality follows from $\lambda=\frac{\tau-2}{\tau-1}$ and $\tau \in(3,5)$.

Again it follows that also the integral converges:

Lemma 4.5 (Convergence of the integral for $\tau \in(3,5)$ ). For $\beta=\beta_{c, N}, B=0$ and $\tau \in(3,5)$,

$$
\lim _{N \rightarrow \infty} \int_{-\infty}^{\infty} \mathrm{e}^{-N G_{N}\left(z / N^{1 /(\tau-1)} ; r\right)} \mathrm{d} z=\int_{-\infty}^{\infty} \mathrm{e}^{z r \sqrt{\frac{\mathbb{E}[W]}{\nu}}-f\left(\sqrt{\frac{\mathbb{E [ W ]}}{\nu}} z\right)} \mathrm{d} z .
$$

Proof. We again start from the rewrite of $G_{N}$ in (4.21). As before,

$$
N \mathbb{E}\left[g\left(W_{N}, z / N^{\frac{1}{\tau-1}}\right)\right]=\sum_{i=1}^{N}\left[\frac{1}{2}\left(\frac{1}{\sqrt{\mathbb{E}\left[W_{N}^{2}\right]}} \frac{c_{w} z}{i^{1 /(\tau-1)}}\right)^{2}-\log \cosh \left(\frac{1}{\sqrt{\mathbb{E}\left[W_{N}^{2}\right]}} \frac{c_{w} z}{i^{1 /(\tau-1)}}\right)\right],
$$

where it is easy to see that the summands are positive and decreasing in $i$. Hence,

$$
N \mathbb{E}\left[g\left(W_{N}, z / N^{\frac{1}{\tau-1}}\right)\right] \geq \int_{1}^{N}\left[\frac{1}{2}\left(\frac{1}{\sqrt{\mathbb{E}\left[W_{N}^{2}\right]}} \frac{c_{w} z}{y^{1 /(\tau-1)}}\right)^{2}-\log \cosh \left(\frac{1}{\sqrt{\mathbb{E}\left[W_{N}^{2}\right]}} \frac{c_{w} z}{y^{1 /(\tau-1)}}\right)\right] \mathrm{d} y .
$$

We want to use (4.24), and hence split the integral in the region where $\left|\frac{1}{\sqrt{\mathbb{E}\left[W_{N}^{2}\right]}} \frac{c_{w} z}{y^{1 /(\tau-1)}}\right|$ is bigger or smaller 
than $A$. This gives

$$
\begin{aligned}
& N \mathbb{E}\left[g\left(W_{N}, z / N^{\frac{1}{\tau-1}}\right)\right] \geq \varepsilon \frac{c_{w}^{2} z^{2}}{\mathbb{E}\left[W_{N}^{2}\right]} \int_{1}^{\left(\frac{A \sqrt{\mathbb{E}\left[W_{N}^{2}\right]}}{c_{w}|z|}\right)^{\tau-1}} \frac{1}{y^{\frac{2}{\tau-1}}} \mathrm{~d} y+\varepsilon \frac{c_{w}^{4} z^{4}}{\mathbb{E}\left[W_{N}^{2}\right]} \int_{\left(\frac{A \sqrt{\mathbb{E}\left[W_{N}^{2}\right]}}{c_{w}|z|}\right)^{\tau-1}}^{N} \frac{1}{y^{\frac{4}{\tau-1}}} \mathrm{~d} y \\
& =\varepsilon \frac{c_{w}^{2} z^{2}}{\mathbb{E}\left[W_{N}^{2}\right]} \frac{\tau-1}{\tau-3}\left(\left(\frac{A \sqrt{\mathbb{E}\left[W_{N}^{2}\right]}}{c_{w}|z|}\right)^{\tau-3}-1\right) \\
& -\varepsilon \frac{c_{w}^{4} z^{4}}{\mathbb{E}\left[W_{N}^{2}\right]} \frac{\tau-1}{5-\tau}\left(N^{-\frac{5-\tau}{\tau-1}}-\left(\frac{A \sqrt{\mathbb{E}\left[W_{N}^{2}\right]}}{c_{w}|z|}\right)^{-(5-\tau)}\right) \\
& =k_{1}|z|^{-(\tau+1)}-k_{2} z^{2}-o(1) z^{4}+k_{3}|z|^{9-\tau},
\end{aligned}
$$

for the proper constants $k_{1}, k_{2}, k_{3}>0$. Since $9-\tau>4$,

$$
\int_{-\infty}^{\infty} \mathrm{e}^{-k_{1}|z|^{-(\tau+1)}+k_{2} z^{2}+o(1) z^{4}-k_{3}|z|^{9-\tau}} \mathrm{d} z<\infty .
$$

Together with the pointwise convergence in the previous lemma, this proves this lemma for $r=0$. For $r \neq 0$, the proof can be adapted as for the case $\mathbb{E}\left[W^{4}\right]<\infty$.

We next analyze the large $x$ behavior of $f(x)$ arising in the density of the limiting random variable:

Lemma 4.6 (Asymptotics of $f$ for $\tau \in(3,5))$. For $\tau \in(3,5)$,

$$
\lim _{x \rightarrow \infty} \frac{f(x)}{x^{\tau-1}}=\left(\frac{\tau-2}{\tau-1}\right)^{\tau-1} \int_{0}^{\infty}\left(\frac{1}{2 y^{2 /(\tau-1)}}-\log \cosh \frac{1}{y^{1 /(\tau-1)}}\right) \mathrm{d} y<\infty .
$$

Proof. We first prove that the integral is finite. For this, define

$$
h(y)=\frac{1}{2} y^{2}-\log \cosh y
$$

so that $h(y) \geq 0$. Then,

$$
\int_{0}^{\infty}\left(\frac{1}{2 y^{2 /(\tau-1)}}-\log \cosh \frac{1}{y^{1 /(\tau-1)}}\right) \mathrm{d} y=\int_{0}^{\infty} h\left(\frac{1}{y^{1 /(\tau-1)}}\right) \mathrm{d} y .
$$

Since $\log \cosh y \geq 0$, we have $h(y) \leq \frac{1}{2} y^{2}$, and hence

$$
h\left(\frac{1}{y^{1 /(\tau-1)}}\right) \leq \frac{1}{2 y^{2 /(\tau-1)}} .
$$

This is integrable for $y \rightarrow 0$, because $2 /(\tau-1)<1$ for $\tau \in(3,5)$.

Using (4.37), for $y$ large,

$$
h\left(\frac{1}{y^{1 /(\tau-1)}}\right) \leq \frac{1}{12} \frac{1}{y^{4 /(\tau-1)}} .
$$

This is integrable for $y \rightarrow \infty$, because $4 /(\tau-1)>1$ for $\tau \in(3,5)$.

To prove that $f(x) / x^{\tau-1}$ converges to the integral as $x \rightarrow \infty$ we rewrite, with $a=(\tau-2) /(\tau-1)$,

$$
\begin{aligned}
\frac{f(x)}{x^{\tau-1}} & =\frac{1}{x^{\tau-1}} \sum_{i=1}^{\infty} h\left(a \frac{x}{i^{1 /(\tau-1)}}\right)=a^{\tau-1} \frac{1}{(a x)^{\tau-1}} \sum_{i=1}^{\infty} h\left(\left(\frac{1}{i /(a x)^{\tau-1}}\right)^{1 /(\tau-1)}\right) \\
& =a^{\tau-1} \int_{0}^{\infty} h\left(\frac{1}{y^{1 /(\tau-1)}}\right) \mathrm{d} y(1+o(1)) .
\end{aligned}
$$




\subsection{Proof of Theorem 2.15}

We can now prove Theorem 2.15 for the measure $\widetilde{P}_{N}$ :

Proof of Theorem 2.15 for the measure $\widetilde{P}_{N}$. We can do a change of variables so that

$$
\int_{-\infty}^{\infty} \mathrm{e}^{-N G_{N}(z ; r)} \mathrm{d} z=N^{1 /(\boldsymbol{\delta}+1)} \int_{-\infty}^{\infty} \mathrm{e}^{-N G_{N}\left(z / N^{1 /(\boldsymbol{\delta}+1)} ; r\right)} \mathrm{d} z
$$

Hence, using Lemma 4.1

$$
\widetilde{P}_{N}\left(\exp \left(r \frac{S_{N}}{N^{\boldsymbol{\delta} /(\boldsymbol{\delta}+1)}}\right)\right)=\frac{\int_{-\infty}^{\infty} \mathrm{e}^{-N G_{N}\left(z / N^{1 /(\boldsymbol{\delta}+1)} ; r\right)} \mathrm{d} z}{\int_{-\infty}^{\infty} \mathrm{e}^{-N G_{N}\left(z / N^{1 /(\boldsymbol{\delta}+1)} ; 0\right)} \mathrm{d} z} .
$$

It follows from Lemma 4.3 for $\mathbb{E}\left[W^{4}\right]<\infty$ and from Lemma 4.5 for $\tau \in(3,5)$ that

$$
\lim _{N \rightarrow \infty} \widetilde{P}_{N}\left(\exp \left(r \frac{S_{N}}{N^{\boldsymbol{\delta} /(\boldsymbol{\delta}+1)}}\right)\right)=\frac{\int_{-\infty}^{\infty} \mathrm{e}^{z r \sqrt{\frac{\mathbb{E}[W]}{\nu}}-f\left(\sqrt{\frac{\mathbb{E}[W]}{\nu}}\right)} \mathrm{d} z}{\int_{-\infty}^{\infty} \mathrm{e}^{-f\left(\sqrt{\frac{\mathbb{E}[W]}{\nu}} z\right)} \mathrm{d} z}=\frac{\int_{-\infty}^{\infty} \mathrm{e}^{x r-f(x)} \mathrm{d} x}{\int_{-\infty}^{\infty} \mathrm{e}^{-f(x)} \mathrm{d} x},
$$

where we made the change of variables $x=\sqrt{\frac{\mathbb{E}[W]}{\nu}} z$ in both integrals to obtain the last equality.

As mentioned, this is sufficient to prove the convergence in distribution of $\frac{S_{N}}{N^{\delta /(\delta+1)}}$ to the random variable $X$ (see [14, Theorem A.8.7(a)]).

For the case $\mathbb{E}\left[W^{4}\right]<\infty$,

$$
\lim _{x \rightarrow \infty} \frac{f(x)}{x^{1+\delta}}=\lim _{x \rightarrow \infty} \frac{\frac{1}{12} \frac{\mathbb{E}\left[W^{4}\right]}{\mathbb{E}[W]^{4}} x^{4}}{x^{4}}=\frac{1}{12} \frac{\mathbb{E}\left[W^{4}\right]}{\mathbb{E}[W]^{4}} .
$$

For $\tau \in(3,5)$, the proof that $\lim _{x \rightarrow \infty} \frac{f(x)}{x^{1+\delta}}=C$ is given in Lemma 4.6.

It remains to show that the statement of Theorem 2.15 also holds for the measure $P_{N}$. This follows from the following lemma:

Lemma 4.7. For $\mathbb{E}\left[W^{4}\right]<\infty$ and $\tau \in(3,5)$,

$$
\lim _{N \rightarrow \infty} P_{N}\left(\exp \left(r \frac{S_{N}}{N^{\lambda}}\right)\right)-\widetilde{P}_{N}\left(\exp \left(r \frac{S_{N}}{N^{\lambda}}\right)\right)=0 .
$$

Proof. As shown in [18],

$$
P_{N}(g(\sigma))=\frac{\sum_{\sigma \in \Omega_{N}} g(\sigma) \mathrm{e}^{\frac{1}{2} \sum_{i, j \in[N]} J_{i j} \sigma_{i} \sigma_{j}}}{\sum_{\sigma \in \Omega_{N}} \mathrm{e}^{\frac{1}{2} \sum_{i, j \in[N]} J_{i j} \sigma_{i} \sigma_{j}}},
$$

where

$$
J_{i j}=\frac{1}{2} \log \left(\frac{e^{\beta} p_{i j}+\left(1-p_{i j}\right)}{e^{-\beta} p_{i j}+\left(1-p_{i j}\right)}\right)=p_{i j} \sinh \beta-p_{i j}^{2} \sinh \beta(\cosh \beta-1)+O\left(p_{i j}^{3}\right),
$$

where we have used the Taylor expansion of $\log (1+x)$ about $x=0$ in the last equality. Hence, using (2.5),

$$
\begin{aligned}
\mathrm{e}^{\frac{1}{2} \sum_{i, j \in[N]} J_{i j} \sigma_{i} \sigma_{j}} & =\mathrm{e}^{\frac{1}{2} \sum_{i, j \in[N]}\left(\left(\frac{w_{i} w_{j}}{\ell_{N}+w_{i} w_{j}}-\frac{w_{i} w_{j}}{\ell_{N}}\right) \sinh \beta-p_{i j}^{2} \sinh \beta(\cosh \beta-1)+O\left(p_{i j}^{3}\right)\right) \sigma_{i} \sigma_{j}} e^{\frac{1}{2} \sinh \beta \sum_{i, j \in[N]} \frac{w_{i} w_{j}}{\ell_{N}} \sigma_{i} \sigma_{j}} \\
& =: \mathrm{e}^{E_{N}(\sigma)} e^{\frac{1}{2} \sinh \beta \sum_{i, j \in[N]} \frac{w_{i} w_{j}}{\ell_{N}} \sigma_{i} \sigma_{j}} .
\end{aligned}
$$


Hence, we can rewrite (4.62) as

$$
P_{N}(g(\sigma))=\frac{\sum_{\sigma \in \Omega_{N}} g(\sigma) \mathrm{e}^{E_{N}(\sigma)} \mathrm{e}^{\frac{1}{2} \sinh \beta \sum_{i, j \in[N]} \frac{w_{i} w_{j}}{\ell_{N}} \sigma_{i} \sigma_{j}}}{\sum_{\sigma \in \Omega_{N}} \mathrm{e}^{E_{N}(\sigma)} \mathrm{e}^{\frac{1}{2} \sinh \beta \sum_{i, j \in[N]} \frac{w_{i} w_{j}}{\ell_{N}} \sigma_{i} \sigma_{j}}}=\frac{\widetilde{P}_{N}\left(g(\sigma) \mathrm{e}^{E_{N}(\sigma)}\right)}{\widetilde{P}_{N}\left(\mathrm{e}^{E_{N}(\sigma)}\right)} .
$$

Combining this with the Cauchy-Schwarz inequality gives

$$
\begin{aligned}
\left|P_{N}\left(\exp \left(r \frac{S_{N}}{N^{\lambda}}\right)\right)-\widetilde{P}_{N}\left(\exp \left(r \frac{S_{N}}{N^{\lambda}}\right)\right)\right| & =\frac{\left|\widetilde{P}_{N}\left(\exp \left(r \frac{S_{N}}{N^{\lambda}}\right)\left(\mathrm{e}^{E_{N}(\sigma)}-\widetilde{P}_{N}\left(\mathrm{e}^{E_{N}(\sigma)}\right)\right)\right)\right|}{\widetilde{P}_{N}\left(\mathrm{e}^{E_{N}(\sigma)}\right)} \\
& \leq \frac{\sqrt{\widetilde{P}_{N}\left(\exp \left(2 r \frac{S_{N}}{N^{\lambda}}\right)\right)} \sqrt{\widetilde{P}_{N}\left(\left(\mathrm{e}^{E_{N}(\sigma)}-\widetilde{P}_{N}\left(\mathrm{e}^{E_{N}(\sigma)}\right)\right)^{2}\right)}}{\widetilde{P}_{N}\left(\mathrm{e}^{E_{N}(\sigma)}\right)} \\
& =\frac{\sqrt{\widetilde{P}_{N}\left(\exp \left(2 r \frac{S_{N}}{N^{\lambda}}\right)\right)} \sqrt{\widetilde{P}_{N}\left(\mathrm{e}^{2 E_{N}(\sigma)}\right)-\widetilde{P}_{N}\left(\mathrm{e}^{E_{N}(\sigma)}\right)^{2}}}{\widetilde{P}_{N}\left(\mathrm{e}^{E_{N}(\sigma)}\right)} .
\end{aligned}
$$

From (4.59), it follows that the first square root converges as $N \rightarrow \infty$. We next analyze $E_{N}(\sigma)$ and show that $E_{N}(\sigma) \rightarrow 0$ in probability w.r.t. $\widetilde{P}_{N}$. We also show that $E_{N}(\sigma)$ is uniformly bounded from above, so that the lemma follows by dominated convergence.

We first analyze the contribution of the $O\left(p_{i j}^{3}\right)$ terms in $E_{N}(\sigma)$. Note that

$$
\sum_{i, j \in[N]} p_{i j}^{3}=\sum_{i, j \in[N]}\left(\frac{w_{i} w_{j}}{\ell_{N}+w_{i} w_{j}}\right)^{3} \leq \sum_{i, j \in[N]}\left(\frac{w_{i} w_{j}}{\ell_{N}}\right)^{3}=\frac{1}{\ell_{N}^{3}}\left(\sum_{i \in[N]} w_{i}^{3}\right)^{2} .
$$

For $\mathbb{E}\left[W_{N}^{2}\right] \rightarrow \mathbb{E}\left[W^{2}\right]<\infty$ it holds that $\max _{i} w_{i}=o(\sqrt{N})$. Hence,

$$
\left(\sum_{i \in[N]} w_{i}^{3}\right)^{2} \leq\left(\max _{i} w_{i}\right)^{2}\left(\sum_{i \in[N]} w_{i}^{2}\right)^{2}=o\left(N^{3}\right) \mathbb{E}\left[W_{N}^{2}\right]^{2}=o\left(\ell_{N}^{3}\right),
$$

because $\ell_{N}=O(N)$. Hence,

$$
\begin{aligned}
E_{N}(\sigma) & =\frac{1}{2} \sum_{i, j \in[N]}\left(\left(\frac{w_{i} w_{j}}{\ell_{N}+w_{i} w_{j}}-\frac{w_{i} w_{j}}{\ell_{N}}\right) \sinh \beta-p_{i j}^{2} \sinh \beta(\cosh \beta-1)+\mathcal{O}\left(p_{i j}^{3}\right)\right) \sigma_{i} \sigma_{j} \\
& =-\frac{1}{2} \sum_{i, j \in[N]}\left(\frac{w_{i}^{2} w_{j}^{2}}{\ell_{N}\left(\ell_{N}+w_{i} w_{j}\right)} \sinh \beta+\left(\frac{w_{i} w_{j}}{\ell_{N}+w_{i} w_{j}}\right)^{2} \sinh \beta(\cosh \beta-1)\right) \sigma_{i} \sigma_{j}+o(1) \\
& =-\frac{1}{2} \sinh \beta \cosh \beta \sum_{i, j \in[N]} \frac{w_{i}^{2} w_{j}^{2}}{\ell_{N}^{2}} \sigma_{i} \sigma_{j}+o(1) \\
& =-\frac{1}{2} \sinh \beta \cosh \beta\left(\sum_{i \in[N]} \frac{w_{i}^{2}}{\ell_{N}} \sigma_{i}\right)^{2}+o(1),
\end{aligned}
$$

where the third equality can be proved as in the analysis of $p_{i j}^{3}$. Hence, $E_{N}(\sigma)$ is indeed uniformly bounded from above, so that $\mathrm{e}^{E_{N}(\sigma)}$ is uniformly bounded. 
It remains to prove that $E_{N}(\sigma) \rightarrow 0$ in probability w.r.t. $\widetilde{P}_{N}$. We define $Y_{N}=\sum_{i \in[N]} \frac{w_{i}^{2}}{\ell_{N}} \sigma_{i}$, so that

$$
E_{N}=-\frac{1}{2} \sinh \beta \cosh \beta Y_{N}^{2}+o(1) .
$$

We analyze the moment generating function of $Y_{N}$ the same way as $S_{N} / N^{\lambda}$. That is, we use the HubbardStratonovich identity to rewrite

$$
\begin{aligned}
& \widetilde{P}_{N}\left(\mathrm{e}^{r Y_{N}}\right)=\frac{\sum_{\sigma \in \Omega_{N}} \mathrm{e}^{r Y_{N}} \mathrm{e}^{\frac{1}{2} \frac{\sinh \beta}{\ell_{N}}\left(\sum_{i \in[N]} w_{i} \sigma_{i}\right)^{2}}}{\sum_{\sigma \in \Omega_{N}} \mathrm{e}^{\frac{1}{2} \frac{\sinh \beta}{\ell_{N}}\left(\sum_{i \in[N]} w_{i} \sigma_{i}\right)^{2}}} \\
& =\frac{\sum_{\sigma \in \Omega_{N}} \mathbb{E}\left[\mathrm{e}^{\left.r \sum_{i \in[N]} \frac{w_{i}^{2}}{N \mathbb{E}\left[W_{N}\right]} \sigma_{i}+\sqrt{\frac{\sinh \beta}{N \mathbb{E}\left[W_{N}\right]}} \sum_{i \in[N]} w_{i} \sigma_{i} Z\right]}\right.}{\sum_{\sigma \in \Omega_{N}} \mathbb{E}\left[e^{\sqrt{\frac{\sinh \beta}{N \mathbb{E}\left[W_{N}\right.}} \sum_{i \in[N]} w_{i} \sigma_{i} Z}\right]} \\
& =\frac{\mathbb{E}\left[\mathrm{e}^{\left.N \mathbb{E}\left[\log \cosh \left(r \frac{W_{N}^{2}}{N \mathbb{E}\left[W_{N}\right]}+\sqrt{\frac{\sinh \beta}{N \mathbb{E}\left[W_{N}\right]}} W_{N} Z\right) \mid Z\right]\right]}\right.}{\mathbb{E}\left[\mathrm{e}^{\left.N \mathbb{E}\left[\log \cosh \left(\sqrt{\frac{\sinh \beta}{N \mathbb{E}\left[W_{N}\right]}} W_{N} Z\right) \mid Z\right]\right]}\right.}
\end{aligned}
$$

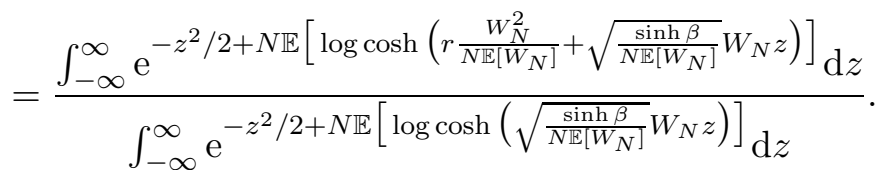

We do a change of variables replacing $z / \sqrt{N}$ by $z$, so that

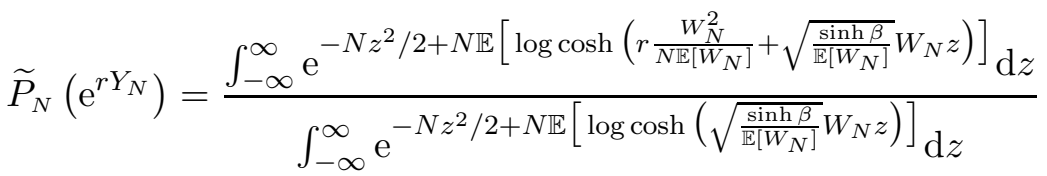

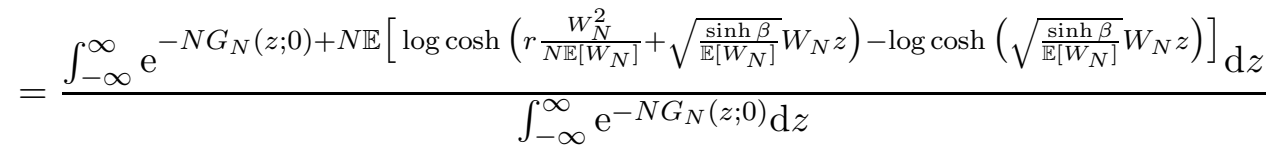

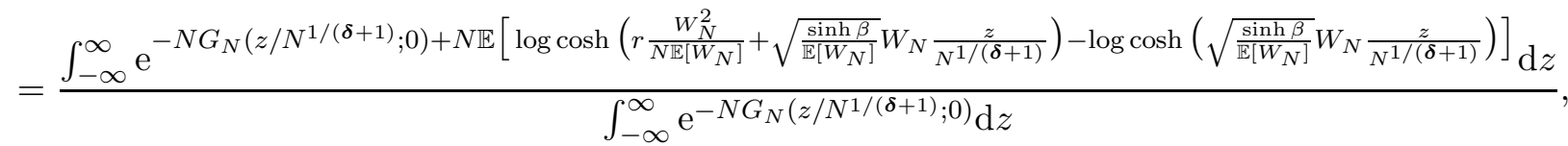

where we did another change of variable in the last equality.

In Lemmas 4.2 and 4.4, we proved that $N G_{N}\left(z / N^{1 /(\boldsymbol{\delta}+1)} ; 0\right)$ converges for $\beta=\beta_{c}$. We Taylor expand the remaining term,

$$
\begin{aligned}
& N \mathbb{E}\left[\log \cosh \left(r \frac{W_{N}^{2}}{N \mathbb{E}\left[W_{N}\right]}+\sqrt{\frac{\sinh \beta}{\mathbb{E}\left[W_{N}\right]}} W_{N} \frac{z}{N^{1 /(\boldsymbol{\delta}+1)}}\right)-\log \cosh \left(\sqrt{\frac{\sinh \beta}{\mathbb{E}\left[W_{N}\right]}} W_{N} \frac{z}{N^{1 /(\boldsymbol{\delta}+1)}}\right)\right] \\
& =\mathbb{E}\left[\tanh \left(\sqrt{\frac{\sinh \beta}{\mathbb{E}\left[W_{N}\right]}} W_{N} \frac{z}{N^{1 /(\boldsymbol{\delta}+1)}}\right) r \frac{W_{N}^{2}}{\mathbb{E}\left[W_{N}\right]}+o(1)\right] .
\end{aligned}
$$

For $\mathbb{E}\left[W_{N}^{3}\right] \rightarrow \mathbb{E}\left[W^{3}\right]<\infty$, which includes power-law distributions with $\tau>4$, we can use that $|\tanh (x)| \leq$ $|x|$, so that

$$
\left|\mathbb{E}\left[\tanh \left(\sqrt{\frac{\sinh \beta}{\mathbb{E}\left[W_{N}\right]}} W_{N} \frac{z}{N^{1 /(\boldsymbol{\delta}+1)}}\right) r \frac{W_{N}^{2}}{\mathbb{E}\left[W_{N}\right]}\right]\right| \leq \sqrt{\frac{\sinh \beta}{\mathbb{E}\left[W_{N}\right]}} \frac{|z r|}{N^{1 /(\boldsymbol{\delta}+1)}} \frac{\mathbb{E}\left[W_{N}^{3}\right]}{\mathbb{E}\left[W_{N}\right]}=o(1) .
$$


For $\tau \in(3,4]$ we use the deterministic choice of the weights as in (2.8) and $\boldsymbol{\delta}=\tau-2$ to rewrite

$$
\begin{aligned}
\mid \mathbb{E}[\tanh ( & \left.\left.\sqrt{\frac{\sinh \beta}{\mathbb{E}\left[W_{N}\right]}} W_{N} \frac{z}{N^{1 /(\boldsymbol{\delta}+1)}}\right) r \frac{W_{N}^{2}}{\mathbb{E}\left[W_{N}\right]}\right]|=| \frac{1}{N} \sum_{i=1}^{N} \tanh \left(\sqrt{\frac{\sinh \beta}{\mathbb{E}\left[W_{N}\right]}} w_{i} \frac{z}{N^{1 /(\tau-1)}}\right) r \frac{w_{i}^{2}}{\mathbb{E}\left[W_{N}\right]} \mid \\
= & \left|\frac{1}{N} \sum_{i=1}^{N} \tanh \left(\sqrt{\frac{\sinh \beta}{\mathbb{E}\left[W_{N}\right]}} \frac{c_{w} z}{i^{1 /(\tau-1)}}\right) \frac{r c_{w}^{2}}{\mathbb{E}\left[W_{N}\right]}\left(\frac{N}{i}\right)^{2 /(\tau-1)}\right| \\
\leq & \frac{|r| c_{w}^{2}}{\mathbb{E}\left[W_{N}\right]} N^{2 /(\tau-1)-1}+N^{2 /(\tau-1)}-1 \sqrt{\frac{\sinh \beta}{\mathbb{E}\left[W_{N}\right]}} \frac{|r z| c_{w}^{3}}{\mathbb{E}\left[W_{N}\right]} \sum_{i=2}^{N} i^{-3 /(\tau-1)} .
\end{aligned}
$$

For $\tau>3$ the first term is $o(1)$. For $\tau \in(3,4)$,

$$
N^{2 /(\tau-1)-1} \sum_{i=2}^{N} i^{-3 /(\tau-1)} \leq N^{-\frac{\tau-3}{\tau-1}} \int_{1}^{N} i^{-3 /(\tau-1)} \mathrm{d} i=\frac{\tau-1}{4-\tau}\left(N^{-\frac{\tau-3}{\tau-1}}-N^{-1 /(\tau-1)}\right)=o(1),
$$

whereas for $\tau=4$

$$
N^{2 /(\tau-1)-1} \sum_{i=2}^{N} i^{-3 /(\tau-1)} \leq N^{-\frac{\tau-3}{\tau-1}} \int_{1}^{N} i^{-3 /(\tau-1)} \mathrm{d} i=N^{-\frac{\tau-3}{\tau-1}} \log N=o(1) .
$$

Hence, in all cases the integrands in the numerator and denominator of (4.72) have the same limit. In Lemmas 4.3 and 4.5 it is proved that the integral in the denominator converges. Since

$$
\left|\mathbb{E}\left[\tanh \left(\sqrt{\frac{\sinh \beta}{\mathbb{E}\left[W_{N}\right]}} W_{N} \frac{z}{N^{1 /(\boldsymbol{\delta}+1)}}\right) r \frac{W_{N}^{2}}{\mathbb{E}\left[W_{N}\right]}\right]\right| \leq \frac{r \mathbb{E}\left[W_{N}^{2}\right]}{\mathbb{E}\left[W_{N}\right]}=O(1),
$$

it follows by dominated convergence that the integral in the numerator has the same limit. Hence,

$$
\lim _{N \rightarrow \infty} \widetilde{P}_{N}\left(\mathrm{e}^{r Y_{N}}\right)=1
$$

from which it follows that $Y_{N} \rightarrow 0$ in probability w.r.t. $\widetilde{P}_{N}$. Hence, also $-\frac{1}{2} \sinh \beta \cosh \beta Y_{N}^{2} \rightarrow 0$ in probability w.r.t. $\widetilde{P}_{N}$. Since $o(1)$ also converges to 0 in probability, so does the sum:

$$
E_{N}=-\frac{1}{2} \sinh \beta \cosh \beta Y_{N}^{2}+o(1) \longrightarrow 0 \quad \text { in probability w.r.t. } \widetilde{P}_{N} .
$$

Remark 4.8 (Sharp asymptotics of the partition function). It follows from the changes of variables in (4.9) and (4.57) that

$$
Z_{N}\left(\beta_{c}, 0\right)=A N^{1 / 2+1 /(\boldsymbol{\delta}+1)} 2^{N}(1+o(1)) .
$$

For $\mathbb{E}\left[W^{4}\right]<\infty$, this exponent equals $1 / 2+1 /(\boldsymbol{\delta}+1)=3 / 4$, whereas for $\tau \in(3,5)$, it is $1 / 2+1 /(\boldsymbol{\delta}+1)=$ $(\tau+1) /(2 \tau-2)$. Thus the partition function has finite-size power-law corrections (in agreement with [6] where the classical Curie-Weiss model is considered). 


\subsection{Scaling window}

Instead of looking at the inverse temperature sequence $\beta_{N}=\beta_{c, N}$ we can also look at $\beta_{N}^{\prime}=\beta_{c, N}+b / N^{\frac{\delta-1}{\delta+1}}$ for some constant $b$. The analysis still works and the limiting density instead becomes

$$
\exp \left\{\frac{b}{2} \cosh \left(\beta_{c}\right) \frac{\mathbb{E}\left[W^{2}\right]^{2}}{\mathbb{E}[W]^{3}} x^{2}-f(x)\right\} .
$$

To see why this is correct we look at the following second moment, which shows up in the expansion of $G_{N}$, see (4.13):

$$
\begin{gathered}
\frac{1}{2} \mathbb{E}\left[\left(\sqrt{\frac{\sinh \left(\beta_{c, N}+b / N^{\frac{\delta-1}{\delta+1}}\right)}{\mathbb{E}\left[W_{N}\right]}} W_{N} \frac{z}{N^{1 /(\boldsymbol{\delta}+1)}}+\frac{r}{N^{\boldsymbol{\delta} /(\boldsymbol{\delta}+1)}}\right)^{2}\right] \\
=\frac{z^{2}}{2 N^{2 /(\boldsymbol{\delta}+1)}} \sinh \left(\beta_{c, N}+b / N^{\frac{\delta-1}{\boldsymbol{\delta}+1}}\right) \frac{\mathbb{E}\left[W_{N}^{2}\right]}{\mathbb{E}\left[W_{N}\right]}+\sqrt{\sinh \left(\beta_{c, N}+b / N^{\frac{\delta-1}{\delta+1}}\right) \mathbb{E}\left[W_{N}\right]} \frac{z r}{N}+o(1 / N) .
\end{gathered}
$$

In the first term, we Taylor expand the sine hyperbolic about $\beta_{c, N}$, which gives

$$
\sinh \left(\beta_{c, N}+b / N^{\frac{\delta-1}{\delta+1}}\right)=\sinh \left(\beta_{c, N}\right)+\cosh \left(\beta_{c, N}\right) b / N^{\frac{\delta-1}{\delta+1}}+O\left(1 / N^{2 \frac{\delta-1}{\delta+1}}\right) .
$$

For the other term, and also for the other terms in the expansion of $G_{N}$, it suffices to note that

$$
\sqrt{\sinh \left(\beta_{c, N}+b / N^{\frac{\delta-1}{\delta+1}}\right)}=\sqrt{\sinh \left(\beta_{c, N}\right)}+O\left(1 / N^{\frac{\delta-1}{\delta+1}}\right) .
$$

Hence, (4.82) equals

$$
\begin{aligned}
& \frac{z^{2}}{2 N^{2 /(\boldsymbol{\delta}+1)}}+\frac{z^{2}}{2 N^{2 /(\boldsymbol{\delta}+1)}} \cosh \left(\beta_{c, N}\right) \frac{b}{N^{\frac{\delta-1}{\delta+1}}} \frac{\mathbb{E}\left[W_{N}^{2}\right]}{\mathbb{E}\left[W_{N}\right]}+\frac{z r}{N} \frac{\mathbb{E}\left[W_{N}\right]}{\sqrt{\mathbb{E}\left[W_{N}^{2}\right]}}+o(1 / N) \\
& =\frac{z^{2}}{2 N^{2 /(\boldsymbol{\delta}+1)}}+\frac{b z^{2}}{2 N} \cosh \left(\beta_{c, N}\right) \frac{\mathbb{E}\left[W_{N}^{2}\right]}{\mathbb{E}\left[W_{N}\right]}+\frac{z r}{N} \frac{\mathbb{E}\left[W_{N}\right]}{\sqrt{\mathbb{E}\left[W_{N}^{2}\right]}}+o(1 / N)
\end{aligned}
$$

In the expansion of $G_{N}\left(z / N^{1 /(\delta+1)} ; r\right)$ the first term in (4.85) drops as usual, whereas the second term in (4.85) remains. After multiplication by $N$ (cf. (4.18)), one has

$$
-N G_{N}\left(z / N^{1 /(\boldsymbol{\delta}+1)} ; r\right)=\frac{b z^{2}}{2} \cosh \left(\beta_{c}\right) \frac{\mathbb{E}\left[W_{N}^{2}\right]}{\mathbb{E}\left[W_{N}\right]}-f\left(\frac{\mathbb{E}\left[W_{N}\right]}{\sqrt{\mathbb{E}\left[W_{N}^{2}\right]}} z\right)+o(1)
$$

Using the substitution $x=\frac{\mathbb{E}\left[W_{N}\right]}{\sqrt{\mathbb{E}\left[W_{N}^{2}\right]}} z$ the above converges in the limit $N \rightarrow \infty$ to the exponent in (4.81), as required.

Limit distribution at $\beta_{c}$ instead of $\beta_{c, N}$. In the above, we look at the inverse temperature sequence $\beta_{N}=\beta_{c, N}$ and then take the limit $N \rightarrow \infty$. Alternatively, we could immediately start with $\beta=\beta_{c}$. The scaling limit that will be seen depends on the speed at which $\nu_{N}$ approaches $\nu$. Indeed, from (2.24) and (2.34), one has $\beta_{c}-\beta_{c, N}=O\left(\nu-\nu_{N}\right)$.

We investigate this for the deterministic weights according to (2.8), and first investigate how close $\nu_{N}$ is to $\nu$. By [3, Lemma 2.2], $\nu_{N}=\nu+\zeta N^{-\eta}+o\left(N^{-\eta}\right)$ with $\eta=(\tau-3) /(\tau-1)$ and $\zeta$ an explicit non-zero constant. Thus, for $\tau>5, \nu_{N}=\nu+o\left(N^{-1 / 2}\right)$. Hence, the results stay the same (see the previous discussion).

When $\tau \in(3,5)$, instead, $\nu_{N}=\nu+\zeta N^{-\eta}+o\left(N^{-\eta}\right)=\nu+\zeta N^{-\frac{\delta-1}{\delta+1}}+o\left(N^{-\frac{\delta-1}{\delta+1}}\right)$, so we are shifted inside the critical window (see the previous discussion). Hence, in this case the limiting distribution changes. 
Table 1: List of symbols used

\begin{tabular}{|c|c|c|}
\hline Symbol & Definition & Description \\
\hline$N$ & & Number of vertices \\
\hline$[N]$ & $\{1, \ldots, N\}$ & Set of first $N$ positive integers \\
\hline$\beta$ & & Inverse temperature \\
\hline$B$ & & External field \\
\hline$H_{N}$ & & Hamiltonian \\
\hline$Z_{N}$ & & Partition function \\
\hline$\phi$ & $\lim _{N \rightarrow \infty} \frac{1}{N} \log Z_{N}$ & Pressure of inhomogeneous Curie-Weiss model \\
\hline$w_{i}$ & & Weight of vertex $i$ \\
\hline$w$ & $\left(w_{1}, \ldots, w_{N}\right)$ & Sequence of weights \\
\hline & $\sum_{i=1}^{N} w_{i}$ & Total weight \\
\hline$G R G_{N}(\boldsymbol{w})$ & & Generalized random graph with weights $\boldsymbol{w}$ and $N$ vertices \\
\hline$p_{i j}$ & $\frac{w_{i} w_{j}}{\ell_{N}+w_{i} w_{j}}$ & Probability of an edge between vertices $i$ and $j$ in $G R G_{N}(\boldsymbol{w})$ \\
\hline$W_{N}$ & & Weight of uniformly chosen vertex \\
\hline$W$ & & Random variable chosen such that $W_{N} \stackrel{\mathcal{D}}{\longrightarrow} W$ \\
\hline$\nu$ & $\mathbb{E}\left[W^{2}\right] / \mathbb{E}[W]$ & Size-biased weight \\
\hline$\nu_{N}$ & $\mathbb{E}\left[W_{N}^{2}\right] / \mathbb{E}\left[W_{N}\right]$ & Its finite volume analogue \\
\hline$\tau$ & & Power-law exponent \\
\hline$Q_{N}$ & & Law of the random graphs \\
\hline$P_{N}$ & & Annealed Ising measure \\
\hline$\psi_{N}$ & $\frac{1}{N} \log Q_{N}\left(Z_{N}\right)$ & Annealed pressure \\
\hline$S_{N}$ & $\sum_{i=1}^{N} \sigma_{i}$ & Total spin \\
\hline$M_{N}$ & $P_{N}\left(S_{N} / N\right)$ & Annealed magnetization \\
\hline & $\frac{\partial}{\partial B} M_{N}$ & Annealed susceptibility \\
\hline$\psi, M, \chi$ & & $\lim _{N \rightarrow \infty}$ of $\psi_{N}, M_{N}, \chi_{N}$, respectively \\
\hline & $\operatorname{asinh}(1 / \nu)$ & Annealed critical inverse temperature \\
\hline$\beta_{c, N}$ & $\operatorname{asinh}\left(1 / \nu_{N}\right)$ & Its finite volume analogue \\
\hline $\boldsymbol{\beta}, \boldsymbol{\delta}, \boldsymbol{\gamma}, \boldsymbol{\gamma}^{\prime}$ & & Critical exponents, see Def. 2.9 \\
\hline$z^{*}$ & & Fixed point of (2.23) \\
\hline$\widetilde{P}_{N}$ & & Curie-Weiss approximation of $P_{N}$, see (4.2) \\
\hline$\widetilde{Z}_{N}$ & & Curie-Weiss approximation of $Z_{N}$, see (4.3) \\
\hline
\end{tabular}


Acknowledgments. We thank Aernout van Enter for helpful discussions on inhomogeneous versions of the CurieWeiss models. We thank Institute Henri Poincaré for the hospitality during the trimester "Disordered systems, random spatial processes and their applications". We acknowledge financial support from the Italian Research Funding Agency (MIUR) through FIRB project grant n. RBFR10N90W. The work of RvdH is supported in part by the Netherlands Organisation for Scientific Research (NWO) through VICI grant 639.033.806 and the Gravitation NeTwORKS grant 024.002.003.

\section{References}

[1] R. Albert and A.-L. Barabási, Statistical mechanics of complex networks. Review of Modern Physics, 74, 47-97 (2002).

[2] I. Benjamini and O. Schramm, Recurrence of distributional limits of finite planar graphs. Selected Works of Oded Schramm, 533-545, Springer New York (2011).

[3] S. Bhamidi, R. van der Hofstad, and J. S. H. van LeeunaARden, Novel scaling limits for critical inhomogeneous random graphs. The Annals of Probability, 40 (6), 299-2361 (2012).

[4] G. Bianconi, Superconductor-insulator transition on annealed complex networks. Physical Review E, 85(6), 061113 (2012).

[5] B. Bollobás, S. Janson, And O. Riordan, The phase transition in inhomogeneous random graphs. Random Structures \& Algorithms, 31(1), 3-122 (2007).

[6] A. Bovier, Statistical mechanics of disordered systems: a mathematical perspective. Cambridge University Press (2006).

[7] F. Comets, N. Gantert And O. Zeitouni, Quenched, annealed and functional large deviations for onedimensional random walk in random environment. Probability Theory and Related Fields, 118(1), 65-114 (2000).

[8] A. Dembo And A. Montanari, Ising models on locally tree-like graphs. Annals of Applied Probability, 20, 565-592 (2010).

[9] A. Dembo And A. Montanari, Gibbs measures and phase transitions on sparse random graphs. Brazilian Journal of Probability and Statistics, 24, 137-211 (2010).

[10] S. Dommers, C. Giardinà And R. van Der Hofstad, Ising models on power-law random graphs. Journal of Statistical Physics, 141(4), 638-660 (2010).

[11] S. Dommers, C. Giardinà And R. van der Hofstad, Ising critical exponents on random trees and graphs. Communications in Mathematical Physics, 328(1), 355-395 (2014).

[12] S.N. Dorogovtsev, A.V. Goltsev And J.F.F. Mendes, Ising models on networks with an arbitrary distribution of connections. Physical Review E, 66, 016104 (2002).

[13] S.N. Dorogovtsev, A.V. Goltsev And J.F.F. Mendes, Critical phenomena in complex networks. Reviews of Modern Physics, 80(4), 1275-1335 (2008).

[14] R.S. ElLIS, Entropy, Large Deviations, and Statistical Mechanics. Springer-Verlag, New York (1985).

[15] R.S. ElLis, C.M. Newman, Limit theorems for sums of dependent random variables occurring in statistical mechanics. Zeitschrift für Wahrscheinlichkeitstheorie und verwandte Gebiete, 44(2), 117-139 (1978).

[16] R.S. Ellis, C.M. Newman, The statistics of Curie-Weiss models. Journal of Statistical Physics, 19(2), 149-161 (1978). 
[17] C. Giardinà, C. Giberti, R. van der Hofstad and M.L. Prioriello, Quenched central limit theorems for the Ising model on random graphs. Journal of Statistical Physics, 160, 1623-1657 (2015).

[18] C. Giardinà, C. Giberti, R. van der Hofstad and M.L. Prioriello, Annealed central limit theorems for the Ising model on random graphs. ALEA, Latin American Journal of Probability and Mathematical Statistics, 13(1), 121-161 (2016).

[19] R. van DeR Hofstad, Random graphs and complex networks. Vol. I. Lecture notes, Preprint, (2014).

[20] M. Krasnytska, B. Berche, Y. Holovatch and R. Kenna, Violation of Lee-Yang circle theorem for Ising phase transitions on complex networks. Europhysics Letters, 111(6), 60009 (2015).

[21] M. Leone, A. Vázquez, A. Vespignani and R. Zecchina, Ferromagnetic ordering in graphs with arbitrary degree distribution. The European Physical Journal B, 28, 191-197 (2002).

[22] A. Montanari, E. Mossel And A. Sly, The weak limit of Ising models on locally tree-like graphs. Probability Theory and Related Fields, 152, 31-51 (2012). 